\title{
Nonlinear Hydrodynamic and Thermoacoustic Oscillations of a Bluff-body Stabilized Turbulent Premixed Flame
}

\author{
Chin Yik Lee ${ }^{\mathrm{a} *}$, Larry Li ${ }^{\mathrm{b} *}$, Matthew Juniper ${ }^{\mathrm{a}}$, and Stewart Cant ${ }^{\mathrm{a}}$ \\ ${ }^{a}$ Department of Engineering, University of Cambridge, Cambridge, UK \\ ${ }^{\mathrm{b}}$ Department of Mechanical and Aerospace Engineering, HKUST, Clear Water Bay, HK
}

( v4.2 released October 2009)

\begin{abstract}
Turbulent premixed flames often experience thermoacoustic instabilities when the combustion heat release rate is in phase with acoustic pressure fluctuations. Linear methods often assume a-priori that oscillations are periodic and occur at a dominant frequency with a fixed amplitude. Such assumptions are not made when using nonlinear analysis. When an oscillation is fully saturated, nonlinear analysis can serve as a useful avenue to reveal flame behaviour far more elaborate than period-one limit cycles, including quasi-periodicity and chaos in hydrodynamically or thermoacoustically self-excited system. In this paper, the behaviour of a bluff-body stabilized turbulent premixed propane/air flame in a model jet-engine afterburner configuration is investigated using computational fluid dynamics (CFD). For the frequencies of interest in this investigation, an unsteady Reynolds-averaged Navier-Stokes (URANS) approach is found to be appropriate. Combustion is represented using a modified laminar flamelet approach with an algebraic closure for the flame surface density. The results are validated by comparison with existing experimental data and with Large-Eddy Simulation (LES), and the observed self-excited oscillations in pressure and heat release are studied using methods derived from dynamical systems theory. A systematic analysis is carried out by increasing the equivalence ratio of the reactant stream supplied to the premixed flame. A strong variation in the global flame structure is observed. The flame exhibits a self-excited hydrodynamic oscillation at low equivalence ratios, becomes steady as the equivalence ratio is increased to intermediate values, and again exhibits a self-excited thermoacoustic oscillation at higher equivalence ratios. Rich nonlinear behaviour including periodic and quasi-periodic oscillations is observed at several different equivalence ratios, and the associated bifurcation points are determined. The investigation demonstrates that turbulent premixed flames can exhibit complex dynamical behaviour including quasiperiodicity, limit cycles, and period-two limit cycles due to the interactions of various physical mechanisms. An understanding of the transition of the turbulent premixed flame from a steady to an oscillatory state allows regimes with strong variation in flame behaviour to be identified. This has implications in selecting the operating conditions for such flames and for devising proper control strategies for the avoidance of thermoacoustic instability.
\end{abstract}

Keywords: turbulent premixed flames; Unsteady Reynolds-averaged Navier-Stokes; Bray-Moss-Libby; Flame surface density; Nonlinear dynamics theory; Thermoacoustic instability

\section{Introduction}

Confined combustion systems are susceptible to thermoacoustic instability, and self-excited oscillations can occur if the pressure fluctuations arising from the natural acoustic modes of the system are in phase with the unsteady heat release [1]. The resultant large amplitude oscillations may cause damage, and restrict the acceptable operating conditions of devices including gas turbines, industrial furnaces,

\footnotetext{
${ }^{*}$ Corresponding author. Email: cyl36@cam.ac.uk

Co author. Email: larryli@ust.hk, mpj1001@cam.ac.uk, rsc10@cam.ac.uk
} 
rocket motors and afterburners. An understanding of the interaction between flowfield or pressure perturbations with the heat release can therefore aid the design of combustion systems to avoid the regimes under which a self-sustained oscillation may be reached [2].

Often, classical linear acoustic theory can provide insights on the frequency and growth rate of an oscillation, and also indicate the possible mode shapes [3, 4]. However, the acoustic oscillation will tend to grow and saturate, and nonlinear interactions can come into play and dominate the flame dynamics once a certain amplitude is exceeded. While flame transfer functions obtained by means of experiments $[5,6]$ or CFD [7-9] can characterize the onset of instability, fewer studies have focused on the effects of saturation $[10,11]$. When an oscillation is fully saturated, nonlinear analysis can serve as a useful avenue to reveal rich flame dynamics and has the potential to predict the amplitude of oscillation. To identify the saturation mechanisms of flames oscillating in limit cycles [12] and to gain a better understanding of processes such as hysteresis, mode switching and triggering $[13,14]$, studies of nonlinear thermoacoustics were carried out recently by solving the nonlinear dispersion relation and using the flame describing functions for different flame configurations $[15,16]$.

Systematic investigation of thermoacoustically oscillating flames is difficult given the complexity of the various mechanisms involved [17], and often these mechanisms are fully coupled when nonlinearity is present. An established approach to investigate the behaviour of these flames is to evaluate the effect of individual parameters on the stability of the system and to identify the presence of Hopf points [18]. Each of these points corresponds to the location of a Hopf bifurcation at which the system becomes unstable to oscillations, and can be identified using linear stability analysis. There are two types of Hopf bifurcation. At a supercritical Hopf bifurcation, the oscillation amplitude grows gradually as a control parameter is changed. At a subcritical Hopf bifurcation, the oscillation amplitude grows abruptly. The sudden appearance of large oscillations may also be observed as a consequence of global bifurcations such as the homoclinic type, which has been identified in diffusion flames [19]. Nonlinear bifurcations of flames have been observed in dump combustors [20], bluff-body stabilized flames [21] and swirl-stabilized combustion chambers [22, 23]. Flame dynamics can change dramatically when the governing parameters, such as flow velocities, swirl number and mixture strength pass through the critical values at which bifurcation points are located. These studies have shown the existence of both subcritical and supercritical Hopf bifurcations in thermoacoustic systems. The subcritical nature of the transition indicates that the system may be bistable, and is capable of nonlinear phenomena such as hysteresis, while triggering due to external disturbances and random noise has also been shown to occur in the subcritical zone $[13,24]$.

One of the key nonlinear components of a thermoacoustic system is the flame itself [25]. Many of the features of combustion instabilities are controlled by the nonlinearities of the flame [26]. Premixed flames can exhibit a series of complex and varied dynamics during thermoacoustic oscillations. The limit-cycle behaviour of a ducted bluff-body turbulent premixed stabilized flame has been investigated numerically [25, 27] and experimentally [21]. Nonlinear bifurcation and dynamical states such as quasi-periodic behaviour of thermoacoustic oscillations were reported in a numerical study in a model combustor [28] and in experiments of a premixed laboratory scale burner [29]. Transition from a steady state to quasiperiodicity and chaos in flames subjected to a variation in burner tip rotation was also observed [30]. More recently, it has been shown [31] that laminar premixed flames can exhibit elaborate nonlinear behaviour during thermoacoustic oscillation, including 
frequency-locked, period-two and chaotic oscillations, when varying the flame position in a Rijke tube. Premixed bluff-body stabilized flames are also susceptible to the hydrodynamic instabilities induced by the Kevin-Helmholtz [32] and/or Bernard/Von-Karman mechanisms [33, 34]. Flow stability from the shear layer to the wake is determined by the interaction between shear generated vorticity and flame generated baroclinic torque [35]. Variation in the vortical structure due to external perturbations can result in frequency-locking and intermittency [36] even with large pockets of local absolute instabilities. The relationship of the forcing frequencies to the global mode frequencies was found to affect the global flame structure. Changes in the flame surface area due to straining and curvature, often associated with intense rollup of the flame caused by large-scale vortices [37-39], can lead to strong perturbations in heat release. This will affect the flame dynamics and can promote thermoacoustic instabilities.

This paper presents a numerical study of the nonlinear hydrodynamic and thermoacoustic oscillations of a wake-stabilized turbulent premixed flame. For this configuration, the flame responds linearly to the perturbation amplitude of velocity oscillation close to the stabilization region, but the flame response decays towards the downstream wake due to kinematic restoration [40]. Similar behaviour has also been observed numerically and suggests the presence of nonlinearities [41]. The current work is motivated by the Volvo afterburner configuration consisting of a triangular bluff-body flameholder set within a rectangular duct $[42,43]$. Combustion instabilities have been observed and reported at equivalence ratios above $\phi=0.80$ in the experiment. Information on the flame behaviour and frequency of oscillation was well-documented, and complex flame dynamics have also been reported [36]. There have also been several CFD studies for this configuration. Steady Reynolds Averaged Navier Stokes (RANS) simulations using the Magnussen-Hjertager combustion model have been performed [43] but showed some discrepancies with experiment in the temperature profiles. Further investigation [44] found that a finer mesh resolution and a two-step reaction mechanism improved the predictions. Unsteady RANS was carried out [45] using a G-equation approach, and the flame stretch was found to have a strong influence on the $\mathrm{CO}$ formation rate, while major species and temperature remained insensitive to flame wrinkling. Large Eddy Simulation (LES) has also been applied for validation of flame-wrinkling and fractal sub-gridscale (SGS) combustion models [46, 47], with focus on how large scale coherent structures modify the flame surface area. Studies also revealed the possibility of symmetrical or asymmetrical vortex shedding, depending on the operating conditions [48]. The flame response to acoustic forcing was also investigated [49], and the reaction rates were found to be overpredicted in highly stretched flame regions induced by large-scale coherent structures. Changes in flame dynamics caused by longitudinal and transverse oscillatory modes at different operating conditions were also studied [47]. More recently, the linear-eddy SGS model has been compared against the eddy-break-up SGS model [50] and shown to be superior in predicting flame spread and mixing rates.

In the present work, the stability of a bluff-body stabilized flame in the Volvo afterburner experiment is studied using URANS with a Reynolds stress turbulence closure together with the Bray-Moss-Libby (BML) combustion model [51] with a modified reaction rate closure [52] that aims to capture the changes in the flame surface density due to straining and curvature effects caused by external flow perturbations. This work builds directly upon the work of Armitage et al. [39] that serves to validate the use of the unsteady RANS to investigate flame instability in the presence of vortex shedding and rollup, and to gain insight into the origins of nonlinear behaviour during forced oscillations. This approach has proved to be 
useful in capturing the response of premixed flames interacting with large-scale coherent flow structures [53]. The primary aim of this work is to provide an insight onto the various dynamical behaviours exhibited by the self-excited turbulent premixed flame. Six different flames are simulated at different equivalence ratios. For each flame, the self-excited response is examined using nonlinear dynamical systems theory, revealing rich behaviour, including quasiperiodicity and period-two oscillations, and the corresponding bifurcations. The flame structure is shown to change dramatically across the bifurcations and a stability map is constructed.

\section{Numerical and modelling framework}

The governing equations for turbulent combustion CFD are the compressible Navier-Stokes equations for mass, momentum, and energy conservation. A Reynolds-averaged approach is taken, using Favre density-weighted ensemble averaging, and a second moment closure model is applied to calculate the turbulent Reynolds stresses [54]. The reaction progress variable is defined as a normalized fuel mass fraction

$$
c=\frac{Y_{F}-Y_{F R}}{Y_{F P}-Y_{F R}}
$$

where $Y_{F}$ denotes the mass fraction of fuel, and the subscripts $R$ and $P$ indicate the reactants and the products, respectively. The local flame properties are determined from the reaction progress variable using the BML flamelet relationships [51] with a heat release parameter that takes into account variations in fuel mass fraction and allows for calculations of flows with partial premixing. The transport equation for the Favre-mean reaction progress variable $\tilde{c}$ is

$$
\frac{\partial \bar{\rho} \tilde{c}}{\partial t}+\frac{\partial \bar{\rho} \tilde{u}_{i} \tilde{c}}{\partial x_{i}}=-\frac{\partial}{\partial x_{i}}\left(\bar{\rho} \widetilde{u_{i}^{\prime \prime} c^{\prime \prime}}\right)+\overline{\dot{\omega}}
$$

The turbulent scalar flux $\widetilde{u_{i}^{\prime \prime} c^{\prime \prime}}$ is modelled using a standard gradient transport hypothesis [55]. The term $\overline{\dot{\omega}}$ is the mean turbulent reaction rate, expressed as the production rate of reaction progress variable per unit volume. This quantity is modelled using a laminar flamelet concept, in which combustion is assumed to take place within thin flame surfaces that separate the regions of reactants and products. Locally, turbulence acts to wrinkle and to distort these interfaces, however, the flame retains the local structure of a strained laminar flame. The reaction rate is given by:

$$
\overline{\dot{\omega}}=\rho_{R} S_{L} I_{0} \Sigma
$$

The group of variables $\rho_{R} S_{L} I_{0}$ represents the reaction rate per unit flame area and $\Sigma$ is the surface area per unit volume. The density of the mixture $\rho_{R}$ is a known quantity. The unstrained laminar flame speed $S_{L}$ is described by an empirical correlation from experimental data from Abu-Orf [56] and takes the form

$$
S_{L}\left(T_{R}, p, \phi\right)=S_{L}^{0}(\phi)\left(\frac{T_{R}}{T_{r e f}}\right)^{\alpha}\left(\frac{p}{p_{r e f}}\right)^{\beta}
$$


where $T_{r e f}$ and $p_{\text {ref }}$ are reference temperature and pressure, respectively. $T_{R}$ is the temperature of the unburnt gas, and $S_{L}^{0}$ is the unstretched laminar flame speed at room temperature and pressure, which is given as a function of equivalence ratio by

$$
S_{L}^{o}(\phi)=a \phi^{b} e^{\left(-c(\phi-d)^{2}\right)}
$$

$\alpha, \beta, a, b, c$ and $d$ in equations (4) and (5) take the value of $1.77,-0.25,0.4243$, $0.7345,4.5003$ and 1.2303 respectively for propane fuel.

The flame surface density (FSD) describes the effects of turbulence on the flame structure, and is modelled using an algebraic expression. The specific form of the model for the flame surface density per unit volume was developed by Brookes et al. [52] and was calibrated against the stagnation-plate flame data of Cheng and Shepherd [57],

$$
\Sigma=\frac{C_{w} \bar{c}^{1.2}\left(1-\bar{c}^{0.8}\right)}{\hat{L}_{y}}
$$

in which the model constant $C_{w}=5.50 \times 10^{-3}$ and $\hat{L}_{y}$ is the integral length scale of flame wrinkling given by:

$$
\hat{L}_{y}=\frac{\nu}{S_{L}}\left(1+C_{f} \frac{u^{\prime}}{S_{L}}\right)^{-1}
$$

where $u^{\prime}$ is the turbulence intensity and $C_{f}$ is taken to be equal to 2.225 .

The correction factor $I_{0}$ accounts for the mean effects of strain and curvature on the laminar flamelet. The influence of curvature is assumed to be negligible since the flame is curved to an equal positive and negative extent on average. The effects of strain rate are modelled following Brookes et al. [52], using data from laminar flame computations with detailed chemistry [58] and Direct Numerical Simulation [59].

The model is implemented in the open source CFD code OpenFOAM [60]. The equations are discretized in space using a central differencing scheme which is second-order accurate in smooth regions of the solution and is flux limited to firstorder near steep gradients in order to guarantee boundedness. First-order bounded Euler-implicit time marching is employed to account for transient effects. The system of equations is solved using the PISO pressure correction algorithm [61]. The algorithm splits the solution procedure into an implicit predictor step followed by two corrector steps. In the predictor step, all quantities except the pressure are updated by solving the momentum, energy and reaction progress variable equation, whilst in the corrector steps, the pressure Poisson equation is solved implicitly and other quantities are updated explicitly.

\section{Configuration and operating conditions}

The configuration corresponds to the Volvo afterburner experiment [42], which consists of a rectangular duct of size $1.0 \mathrm{~m}$ (length) $\times 0.12 \mathrm{~m}$ (height) $\times 0.24 \mathrm{~m}$ (width) containing an equilateral triangular bluff body of side $0.04 \mathrm{~m}$ with one apex pointing into the reactant stream, $0.318 \mathrm{~m}$ downstream from the inlet. A two-dimensional CFD domain is considered, and is divided into ten mesh blocks 
as shown in Fig. 1. The mesh cell count is $280 \times 54$ for blocks 1 and $2 ; 40 \times 54$ for blocks 3 and $4 ; 350 \times 54$ for blocks 5,6 and $7 ; 350 \times 54$ for blocks 8,9 and 10. The mesh spacing around the bluff-body (blocks 3-7) is refined to capture the local flame structure near the stabilization point. Mesh independence is achieved, since no discernible difference in the solution was observed after either doubling or halving the number of mesh points in each direction.

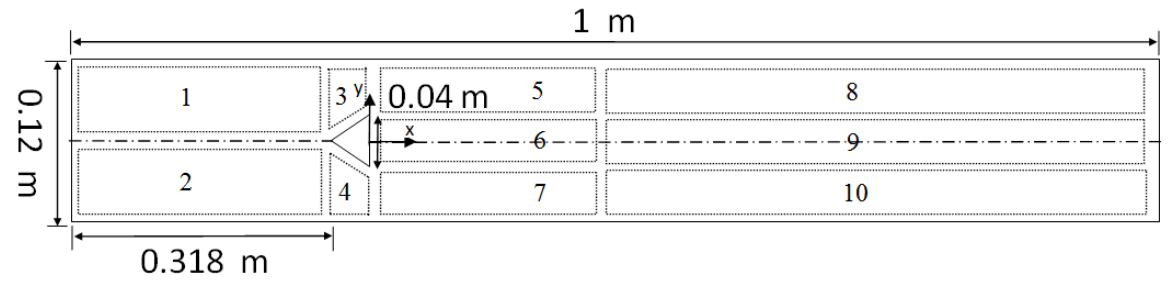

Figure 1.: Volvo afterburner geometry (not to scale)

The inflow axial velocity of the fully premixed propane-air mixture was fixed at $17.3 \mathrm{~m} / \mathrm{s}$ with $4 \%$ turbulence intensity, based on the measured inlet data. The turbulence dissipation rate at the inlet is obtained following Johansson [62]. The inlet temperature is $288 \mathrm{~K}$, and a partially reflecting boundary close to the atmospheric pressure at $101325 \mathrm{~Pa}$ is applied at the outlet. A zero gradient is specified for all other variables at the boundary. The walls are treated as rigid, stationary, adiabatic and impermeable, and standard turbulence wall functions are used.

The objective of the study is to demonstrate the nonlinear behaviour of the system by using the inlet equivalence ratio as the control parameter with other variables kept fixed. One nonreacting and six reacting simulations were carried out, as summarized in Table 1. To extract data for nonlinear analysis, the simulations are continued until a steady/periodic solution is achieved, and flow statistics are collected for a minimum of 50 flow-through times. Self-excitation occurs naturally in the simulations without imposing any external perturbations.

\begin{tabular}{|cc|}
\hline Case & equivalence ratio \\
\hline 1 (cold validation) & 0.0 \\
2 (hot validation) & 0.65 \\
3 (hot) & 0.60 \\
4 (hot) & 0.70 \\
5 (hot) & 0.75 \\
6 (hot) & 0.80 \\
7 (hot) & 0.85 \\
\hline
\end{tabular}

Table 1: Conditions for numerical simulation

\section{Dynamical Systems Theory}

A dynamical system is a system that evolves in time, and can be described by its state. The state of a dynamical system is determined by its dynamical variables and evolves according to the equations of motion that govern the causal relation between the present and future states. In this study, the ducted turbulent premixed 
flame is treated as a dynamical system. A qualitative change in the behaviour exhibited by the flame when changing the control parameter (mixture strength) is known as a bifurcation. When the control parameter moves outside a linearly stable regime, the dynamical system transitions from a steady state without oscillation to a state with oscillation. The operating point at which the dynamical system transitions from a steady to an oscillating state is called a Hopf point.

Phase space contains all possible dynamical states of a system. The asymptotic state of a nonlinear system forms a well-defined geometrical structure in the phase space known as the attractor. Each point contained in the phase space corresponds to a unique state of the dynamical system at a particular time. If there are $m$ dynamical variables, then the state at a given time can be represented by a point in the Euclidean space $\mathbb{R}^{m}$. The representative points trace out a path in the phase space, known as the phase trajectory. To construct the phase space, it is necessary to know the dimension of the phase space, or the degree of freedom (DOF) for a particular system, which is generally given by the number of the coupled set of ordinary differential equations (ODE). This quantity is often not known a-priori and therefore has to be estimated. The embedding theorem proposed by Takens [63] allows the possibility of reconstruction of a phase space from scalar measurements obtained in experiments or CFD. This method asserts that if a time series is one component of an attractor that can be represented by a smooth $d$-dimensional manifold then the topological properties of the attractor are equivalent to the topological properties of the embedding formed by the $m$-dimensional phase space vectors.

$$
\begin{array}{r}
\vec{X}_{i}=(y(i \Delta t), y(i \Delta t+\tau), y(i \Delta t+2 \tau) \\
\ldots, y(i \Delta t+(m-1) \tau))
\end{array}
$$

where $m$ is the embedding dimension and $\tau$ is the delay time. The variable $y$ takes the form of $p^{\prime}$ in this study as we are interested in computing the evolution of the pressure fluctuations. For a proper reconstruction, it is important to calculate the optimum delay time and the optimum embedding dimension. The minimum value of $m$ required to capture the topological properties of an attractor depends on the dimension of the attractor. The delay time has to be chosen carefully: reconstructions with high correlations between consecutive phase space points will result if the delay times are too small; the deterministic structure linking points along a single degree of freedom may be obscured for very large delays. Using the method proposed by Abarbanel [64], the optimal delay time is obtained by computing the first-zero crossing of the autocorrelation function.

The phase portrait is employed to visualize the reconstructed phase space. It is a three-dimensional plot of the system motion against that same motion shifted by one delay time and by two delay times. The topological manifold of the phase trajectory provides valuable information about the system dynamics. When the system is steady, a single point will be observed if no noise is present. For a limit cycle, the attractor is a closed curve, indicating that the solution is periodic in nature. For a quasiperiodic oscillation, two incommensurate frequencies are present, which modulate the amplitude of the solutions. As a result, the attractor will take the form of a torus in the phase space.

The topological dimension of an attractor is given by its correlation dimension. Like other fractal dimensions, it is used to identify the underlying dynamics of a system. By considering a simple Euclidean geometry, points have dimension 0, lines have dimension 1, plane surfaces have dimension 2, solids have dimension 3. Analogously in the examination of the dynamical system, the correlation dimension 
is zero for a steady flow (fixed point), one for a periodic limit cycle (closed trajectory) and two for quasiperiodicity (torus surface), fractional for chaotic motion (self-similar fractal object), and infinite for purely random noise.

\section{Results}

\subsection{Validation}

Before considering the thermoacoustic behaviour of the flame, validation studies for the non-reacting and reacting flow were performed using the experimental data $[43,65]$. Both 2D and 3D URANS simulations are conducted to compare and assess the accuracy of these two approaches. These results are used to provide both a preliminary evaluation of the fundamental flow features and a quantitative indication of the ability of the URANS approach to represent the flow field and global flame structure.

(a)

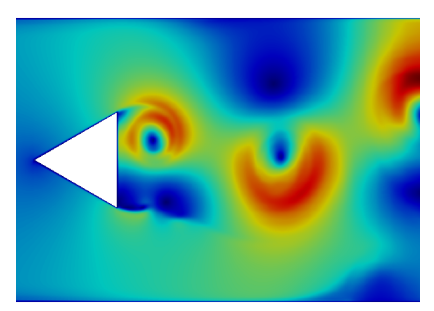

(c)

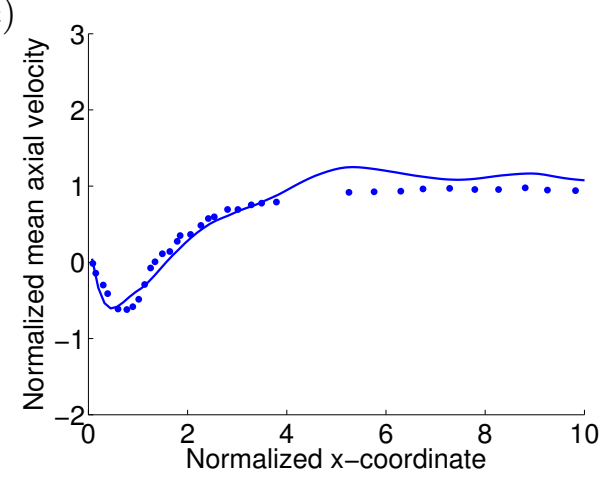

(b)
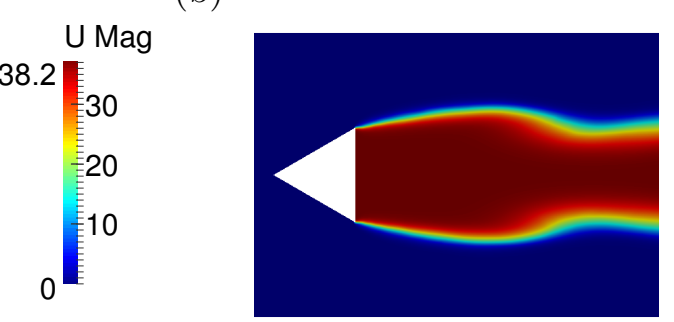

T

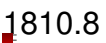

1600

1200

800

400

287.6

(d)

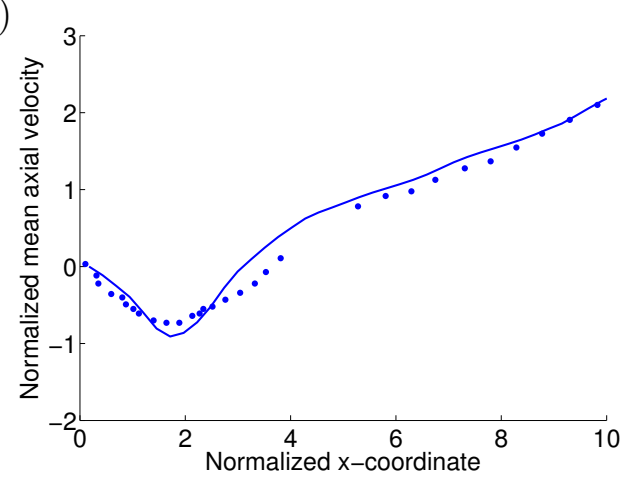

Figure 2.: Flow features for non-reacting flow (a) and reacting flow (b) for 2D case. Centreline time-averaged axial velocity profiles downstream of the bluff-body flameholder for non-reacting flow (c) and reacting flow (d). For (c) and (d), points: experimental data and blue lines: numerical results for $2 \mathrm{D}$ simulation

For the non-reacting 2D case, a snapshot of the instantaneous velocity field and the time-averaged axial velocity along the centreline are plotted in Fig. 2(a) and Fig. 2(c), respectively. For the 2D reacting case, a snapshot of the instantaneous temperature field is shown in Fig. 2(b) and the centreline time-averaged axial velocity is depicted in Fig. 2(d). Values for the time-averaged axial velocity and centreline distance are normalized against the inlet velocity and the width of the bluff-body flameholder $w_{f}$. The results of the $3 \mathrm{D}$ case are presented in Fig. 3 in the same arrangement. The same modelling approach and boundary conditions as used for the $2 \mathrm{D}$ simulation are also used for the full $3 \mathrm{D}$ simulation. The geometry 
for the 3D case includes the spanwise direction and has dimensions that match the Volvo experimental rig. The boundaries at both ends of the spanwise direction are treated as adiabatic walls. The mesh count for the 3D computational domain is 5 million cells, and the mesh resolution is found to be adequate to satisfy mesh independence.

(a)

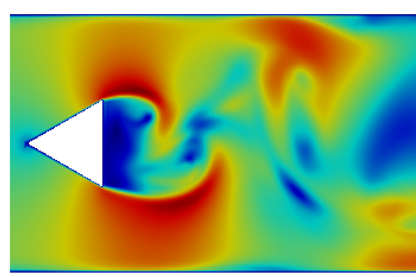

(c)

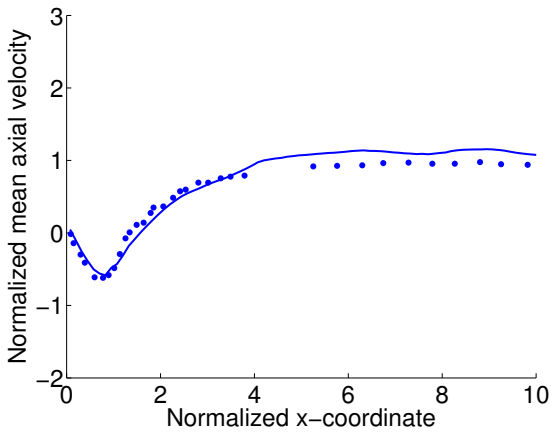

(b)

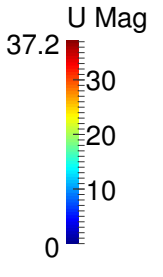

(d)
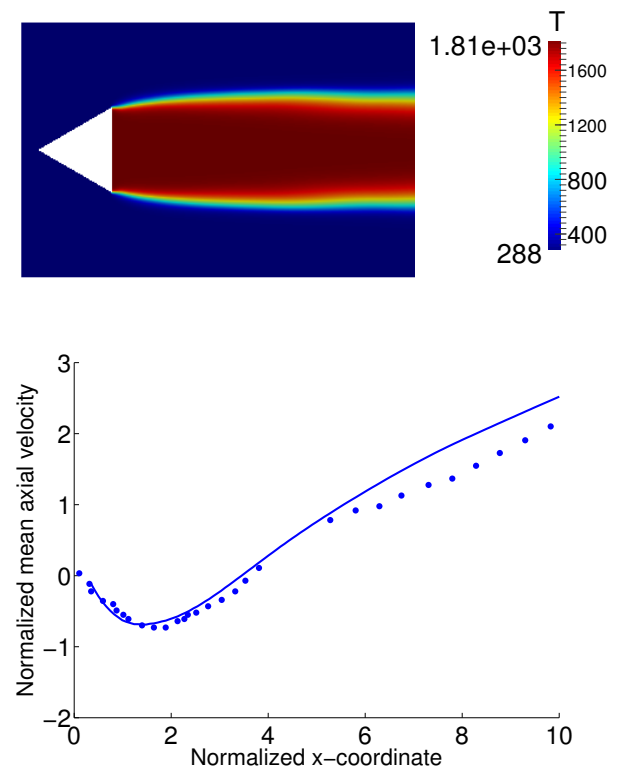

Figure 3.: Flow features for non-reacting flow (a) and reacting flow (b) for 3D case. Centreline time-averaged axial velocity profiles downstream of the bluff-body flameholder for non-reacting flow (c) and reacting flow (d). For (c) and (d), points: experimental data and blue lines: numerical results for $3 \mathrm{D}$ simulation

In Fig. 2(a) and Fig. 3(a), the Karman vortex street caused by the two shear layers formed at the trailing edge of the flameholder is well represented. Alternating roll-up of the these vortex sheets occurs close to the flameholder in the recirculation region. A difference between the $2 \mathrm{D}$ and $3 \mathrm{D}$ result for the cold flow is found in the downstream region. In the 2D case (Fig. 2(a)), vortex breakdown is inhibited further downstream as the spanwise variations in velocity due to vortex stretching effects are not accounted for. In the 3D case (Fig. 3(a)), however, breakdown of vortices is captured. The flame in Fig. 2(b) is represented by a steep gradient of the temperature across the flame front, and can be visualized as a sheet-like structure emanating from the upper and lower edges of the flameholder. The flame lies almost parallel to the separating flow introducing a baroclinic torque which will tend the counter the effect of the fluid-mechanical rotation due to vortex shedding. This suppresses the Karman-type instability that is present in the non-reacting flow, resulting in a symmetrical flame structure. The shape of the mode changes from the non-reacting to reacting case. For the nonreacting case, it is a sinuous (flapping) motion while for the reacting case, it is a varicose (bulging) motion. This is visible in Figs. 2 (a) and 2(b). Both these types of behaviour are observed in the Volvo afterburner experiment [42]. The prediction of the recirculation zone length (location of the dip in the time-averaged axial velocity along the centreline) and the magnitude of the normalized axial velocity in both the non-reacting and 
reacting flow are also in good agreement with experimental data, as shown in Fig. 2(c) and Fig. 2(d). An increase in the recirculation zone length from 1.5 $w_{f}$ in the nonreacting condition to $2 w_{f}$ in the reacting condition is evident. This is associated with volumetric expansion caused by chemical heat release.

(a)

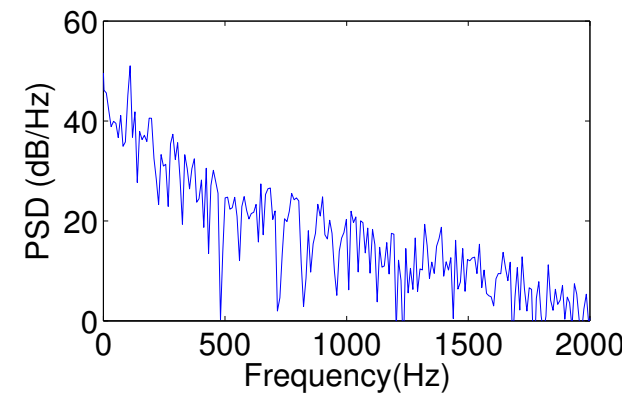

(c)

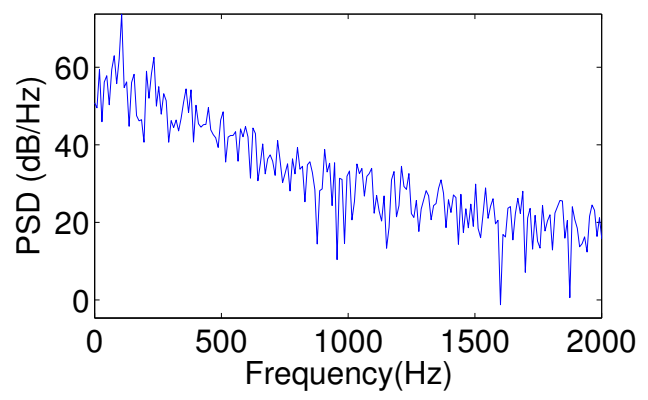

(b)

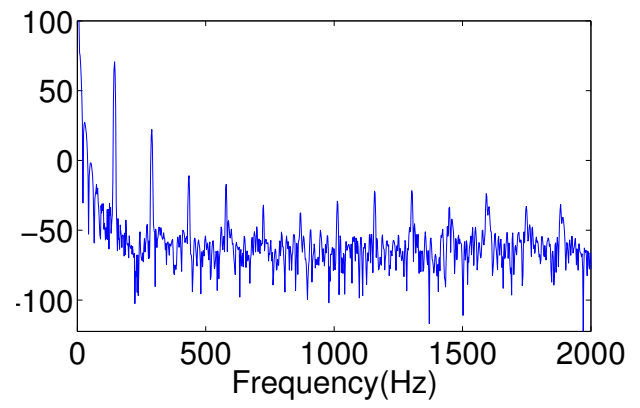

(d)

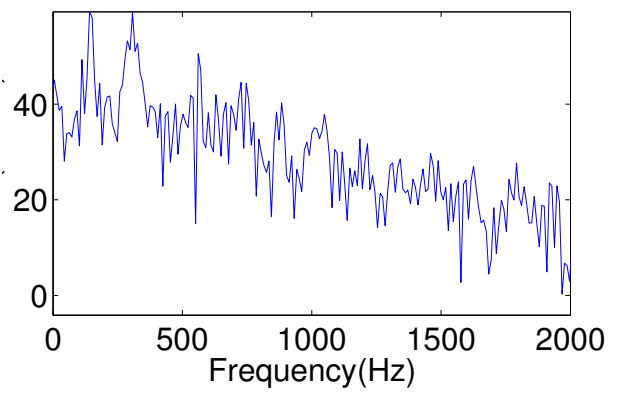

Figure 4.: PSD for 2D non-reacting flow (a), 2D reacting flow (b), 3D non reacting flow (c) and $3 \mathrm{D}$ reacting flow $(\mathrm{d})$

The Power Spectral Density (PSD) of the velocity signal taken at a location within the shear layer downstream of the recirculation zone $(\mathrm{x}=0.35 \mathrm{~m}$ and $\mathrm{y}=$ $0.06 \mathrm{~m}$ ) is shown in Fig. 4(a) for the 2D non-reacting case and Fig. 4(b) for the 2D reacting case. A base unit for velocity at $1 \mathrm{~m} / \mathrm{s}$ is used as the reference value for $\mathrm{dB}$ conversion. The PSD of the 3D case for the velocity signal taken at the same location is shown in Fig. 4(c) for the non-reacting case and Fig. 4(d) for the reacting case. A spectral peak at $107 \mathrm{~Hz}$ is present in the PSD of the non reacting flow. The frequency corresponds to the frequency of the vortex shedding. The Strouhal number of the oscillation $\mathrm{St}=f w_{f} / u_{\text {inlet }}$ based on the bluff body flameholder width of $w_{f}=0.04 \mathrm{~m}$ and inlet velocity of $u_{\text {inlet }}=17.3 \mathrm{~m} / \mathrm{s}$ is approximately 0.251 . This value compares well against the experimental Strouhal number of 0.25. An increase in frequency is evident when combustion is present, where the peak frequency shifts to $144 \mathrm{~Hz}$ and corresponds to a Strouhal number of 0.32. This frequency is in agreement with previous LES studies in which Strouhal number has been reported to be in the range $\mathrm{St}=0.30-0.32[50,66]$. As shown in Fig. 4(c) and Fig. 4(d), the frequencies obtained in the 3D simulation for both the non-reacting and reacting cases are similar to those obtained in the $2 \mathrm{D}$ simulations. It can be seen that the PSD amplitude in the 3D case is higher than that in the 2D case. This is caused by the dimensionality of the problem. Inclusion of the third dimension in the CFD leads to changes in the grid arrangement, while additional boundaries are 
imposed on both ends of the spanwise direction. These factors lead to differences in the numerical solution. Physical differences such as spanwise variation in the flow in the 3D case can also to changes in the momentum transport especially near the shear layer region.

A comparison of the time-averaged axial and transverse velocity profiles for the non-reacting and reacting flow at 5 different axial locations corresponding to 0.375 $w_{f}, 0.95 w_{f}, 1.5 w_{f}, 3.75 w_{f}$ and $9.4 w_{f}$ are depicted in Fig. 5 and Fig. 6, respectively. The time-averaged axial velocities are shown in the top row and the time-averaged transverse velocities are illustrated in the bottom row. Both the axial and transverse velocities are normalized against the inlet velocity. The points represent experimental data, the blue lines denote the numerical results for the $2 \mathrm{D}$ simulation and the red lines denote the numerical results for the 3D simulation.

(a)

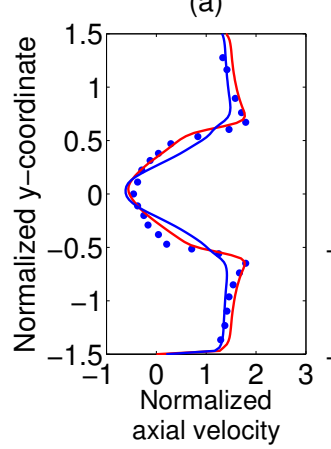

(f)

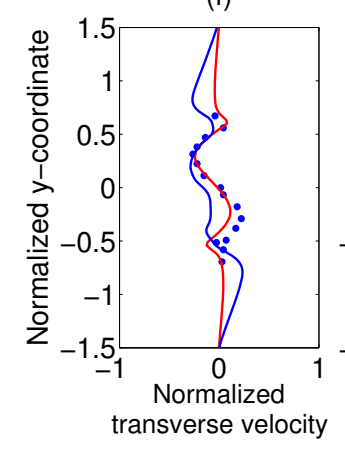

(b)

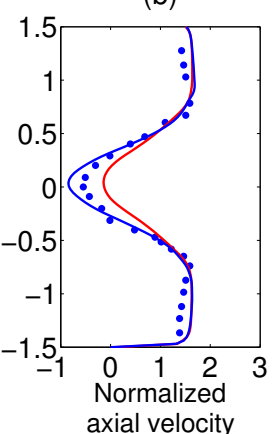

(g)

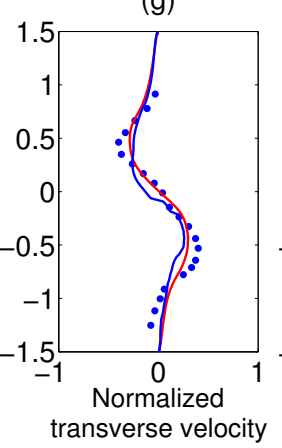

(c)

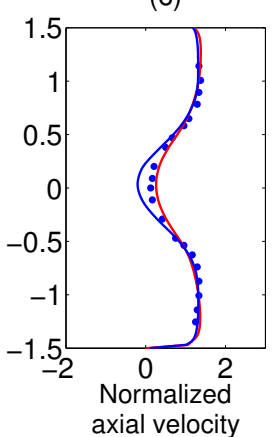

(h)

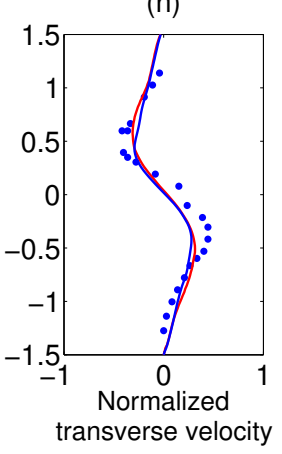

(d)

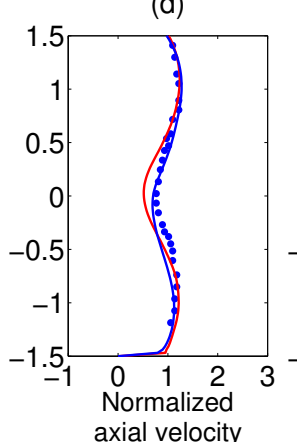

(i)

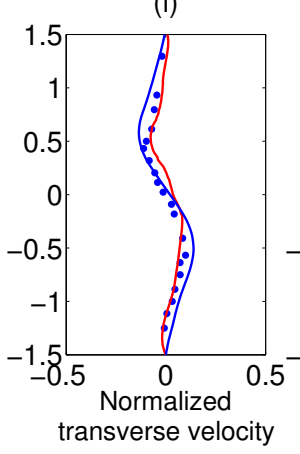

(e)

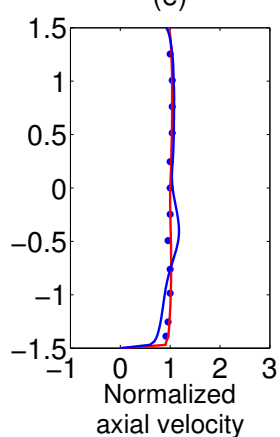

(j)

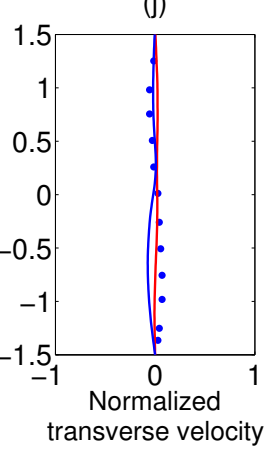

Figure 5.: Normalized axial (a-e) and transverse velocity (f-j) for the non-reacting flow at $0.375 w_{f}, 0.95 w_{f}, 1.5 w_{f}, 3.75 w_{f}$ and $9.4 w_{f}$. Points: experimental data, blue lines: numerical results for $2 \mathrm{D}$ simulation and red lines: numerical results for $3 \mathrm{D}$ simulation

Reasonable agreement between the numerical and experimental values is obtained for both the cold and reacting flow. For the cold flow, the time-averaged axial velocity in Figs. 5(a-b) shows that a strong flow reversal is present immediately behind the bluff-body flameholder. Variations of mean transverse velocity caused by vortex shedding are also present as distinct asymmetrical peaks can be seen on either side of the centreline in Fig. 5(f-i). The intensities of both the mean axial and transverse velocities remain high over a distance from the flamholder of approximately $1.5 w_{f}$. The strength of the adverse velocity gradient reduces downstream at $9.4 w_{f}$ (Fig. 5(e)), where the axial velocity becomes more uniform. The mean transverse velocity also decreases after its peak value at $1.5 w_{f}$ as seen in Fig. 5(h). 
(a)

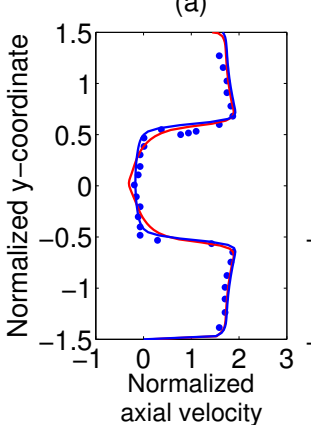

(f)

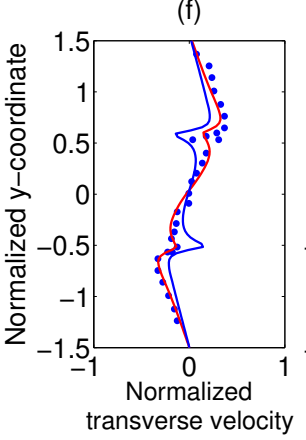

(b)

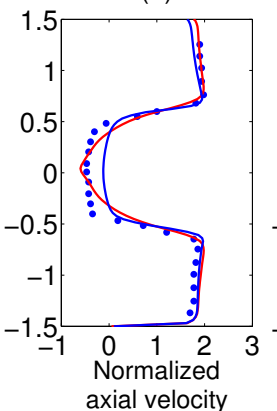

(g) (c)

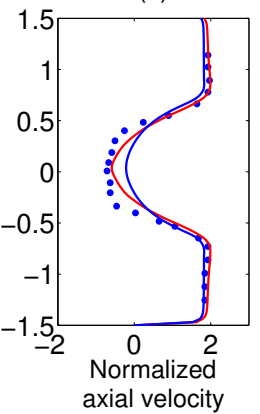

(h) (d)

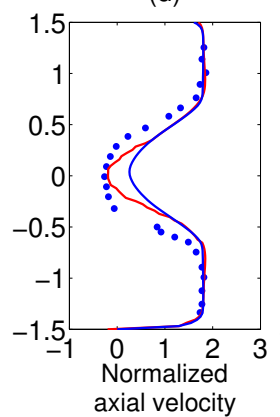

(i) (e)

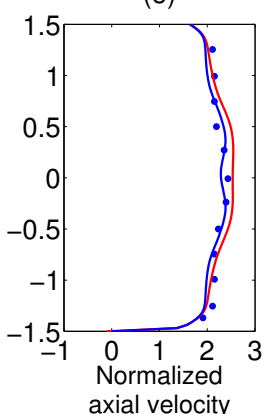

(j)
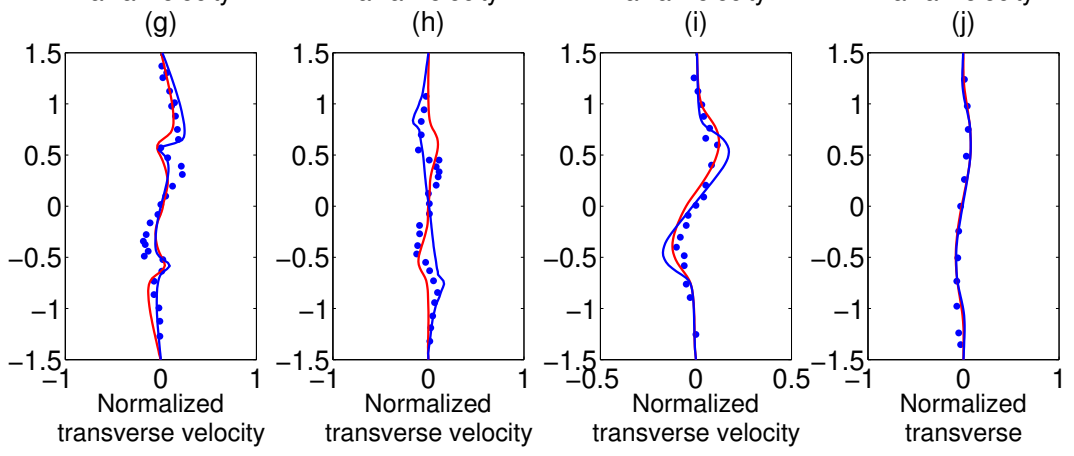

Figure 6.: Normalized axial (a-e) and transverse velocity (f-j) for the reacting flow at $0.375 w_{f}, 0.95 w_{f}, 1.5 w_{f}, 3.75 w_{f}$ and $9.4 w_{f}$. Points: experimental data, blue lines: numerical results for $2 \mathrm{D}$ simulation and red lines: numerical results for $3 \mathrm{D}$ simulation

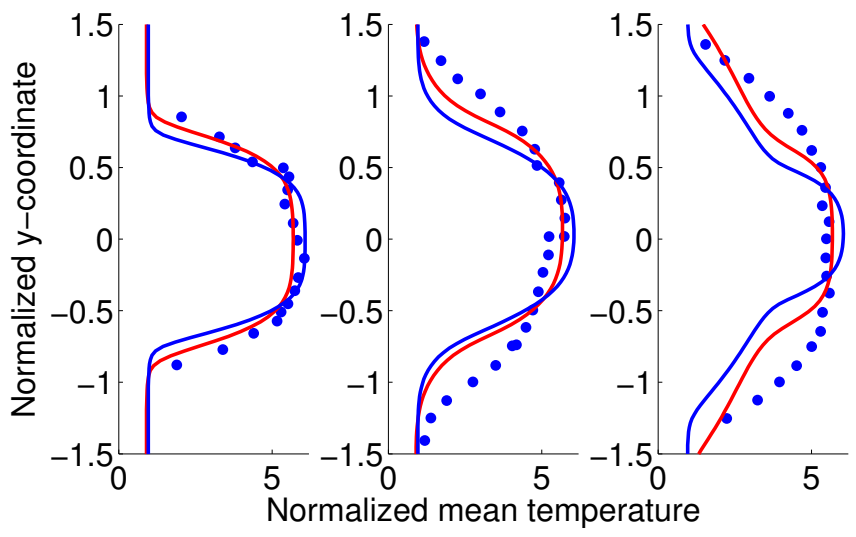

Figure 7.: Normalized temperature profile at $3.75 w_{f}, 8.75 w_{f}$ and $13.75 w_{f}$. Points: experimental data, blue lines: numerical results for $2 \mathrm{D}$ simulation and red lines: numerical results for 3D simulation

For the reacting flow, the normalized time-averaged axial velocity indicates that the recirculation zone remains prominent up to a downstream distance of 3.75 $w_{f}$ (Fig. 6(d)). The axial velocity in the downstream region is almost 2.5 times larger than the inlet flow velocity as a result of the heat release in the wake, as shown in Fig. 6(e). The region of flow reversal (width of the low axial velocity region) is also wider than in the non-reacting case. In Figs. 6(f-i), the magnitudes 
of the normalized time-averaged transverse velocity are much lower than in the non-reacting flow. This is due to thermal expansion and baroclinicity which create a stabilizing effect on the hydrodynamics of the flame. Another notable aspect of the normalized transverse velocity is the inflection point in the shear layers around the flame stabilization region. This can be seen in the region of $\mathrm{y}=0.5$ and $\mathrm{y}=$ -0.5 in Fig. $6(\mathrm{f})$ and $(\mathrm{g})$. This behaviour corresponds to the rapid change in velocity across the flame front, which does not exist under non-reacting conditions. This effect dissipates further downstream.

Results for the mean temperature profile for the reacting flow at three locations $3.75 w_{f}, 8.75 w_{f}$ and $13.75 w_{f}$ are depicted in Fig. 7. The temperature is normalized by the inlet temperature and the distance is normalized by the bluff body width. The normalized temperature field in the vicinity of the flameholder $3.75 w_{f}$ matches quite well with experimental data. Further downstream, the spreading rate of the time-averaged temperature profile is underestimated.

Results for the time-averaged velocities and temperature for the 3D case are denoted by the red lines in Fig. 6 and Fig. 7 respectively. In general, the 3D results offer a slight improvement in the agreement with experimental data for both velocities and temperature profiles at different axial locations. Visually, the $3 \mathrm{D}$ simulation results appear almost identical to the $2 \mathrm{D}$ simulation results extended in the spanwise direction. Despite the slightly better agreement, the computational cost for the unsteady 3D simulation (to solve for Reynolds stresses) is significantly higher and the improvement in the results does not justify its use for the present investigation. Further, for the cases of interest in this study, large-scale coherent motion perturbing the flame is expected to occur in the longitudinal direction, and transversal variation in the flow structure is assumed to be stochastic instead of deterministic in nature.
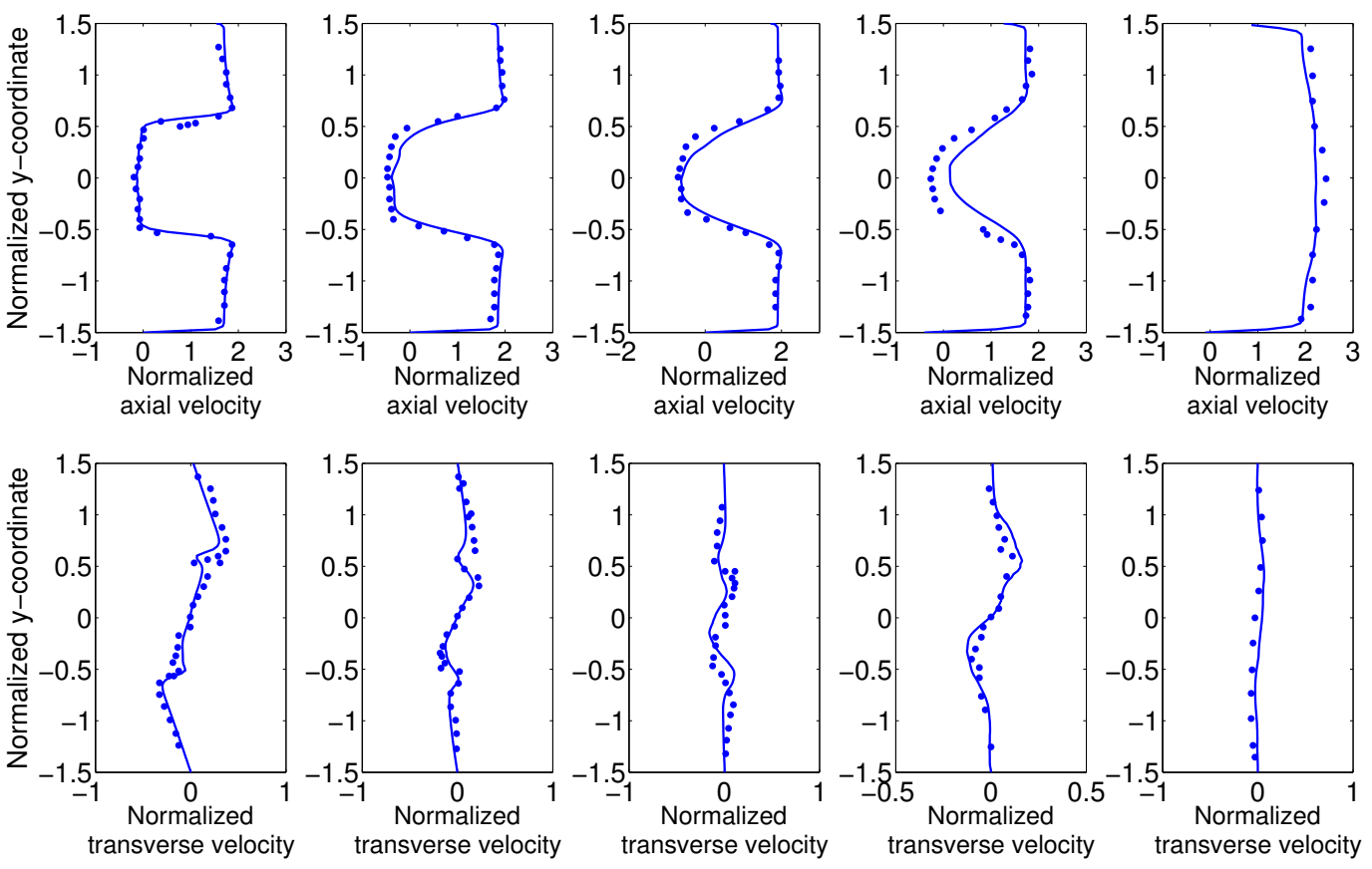

Figure 8.: Normalized axial (a-e) and transverse velocity (f-j) for the reacting flow at $0.375 w_{f}, 0.95 w_{f}, 1.5 w_{f}, 3.75 w_{f}$ and $9.4 w_{f}$. Points: experimental data, blue lines: numerical results for LES 

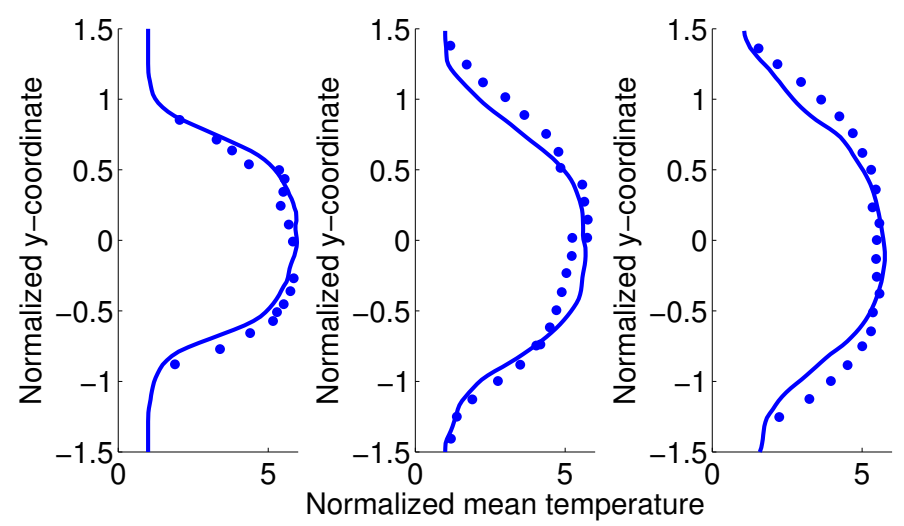

Figure 9.: Normalized temperature profile at $3.75 w_{f}, 8.75 w_{f}$ and $13.75 w_{f}$. Points: experimental data, blue lines: numerical results for LES
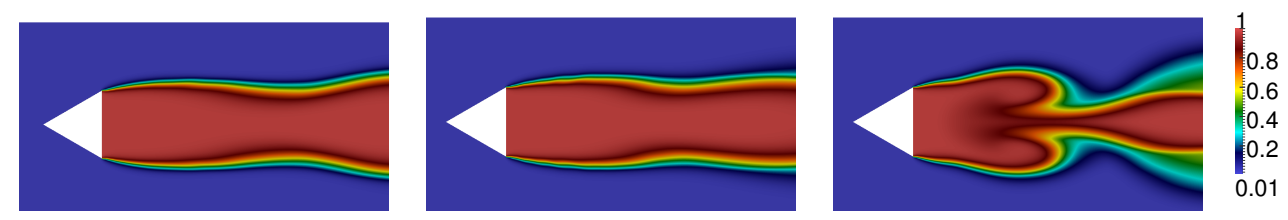

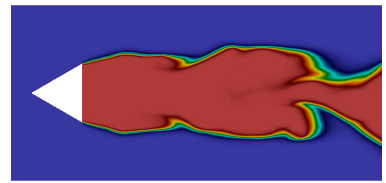

(a)

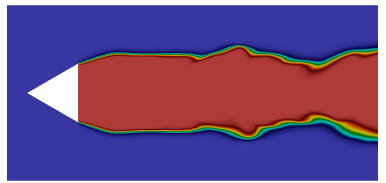

(b)

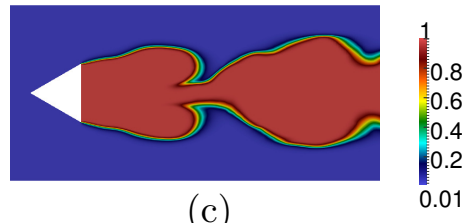

(c)

Figure 10.: Reaction progress variable for URANS (top) and LES (bottom) at $\phi=$ 0.65 (a), $\phi=0.75$ (b) and $\phi=0.85$ (c).

Further validation has been carried out using LES with a flamelet-based combustion model on a mesh of 8 million cells [67] in order to demonstrate that the URANS approach is able to capture the hydrodynamic oscillation. The time-averaged velocity profiles and temperature profiles from the LES are shown in Fig. 8 and Fig. 9, respectively. It is clear that good agreement is obtained between the LES and experimental data, and indeed the level of agreement is comparable with the 2D and 3D URANS results shown in Figs. 6 and Fig. 7. Figure 10 shows a like-forlike comparison of the instantaneous reaction progress variable fields between $3 \mathrm{D}$ URANS and LES for $\phi=0.65,0.75$ and 0.85 . The results suggest that the temporally periodic flame rollup phenomenon observed in the LES can also be captured in URANS. Hence, having validated the numerical results against experiment and against each other, and taking note of the relative computational expense for the number of oscillation cycles required by the nonlinear analysis method, we choose to adopt the 2D URANS approach. The use of URANS implies some additional considerations. Firstly, the presence of external (natural) forcing, in which acoustic oscillation acts as a background forcing to a system exhibiting inherent hydrodynamic instabilities, is assumed not to affect the turbulence closure modelling [40]. Secondly, the extent of far-field flame wrinkling is expected to be smoothed out 
somewhat due to ensemble averaging [68]. This may affect the amplitude of the oscillation, but statistically coherent motion such as vortex shedding and large-scale flame rollup will be retained [69], as confirmed by the present validation study.

\subsection{Effect of Equivalence Ratio on Bifurcation}

The investigation described in this section will primarily focus on the systematic characterization of the nonlinear flame dynamics during the saturated stage of the oscillation, where the key mechanisms governing the global flame behaviour are caused by vortices formed pass the bluff body that serve to affect the self-excited flame dynamics.

Figure 11 shows the normalized pressure (ratio of mean value to the atmospheric pressure at $101325 \mathrm{~Pa}$ ) against the mixture strength. The pressures from the simulations are taken at three different locations, at $0.325 \mathrm{~m}, 0.4 \mathrm{~m}$ and $0.5 \mathrm{~m}$ respectively downstream of the flameholder. It is found that the value of the normalized mean pressure increases as the mixture strength is increased. The value is also seen to be essentially invariant with spatial location, thus indicating the consistency in behaviour in different regions. The increase in pressure with the equivalence ratio was also observed in the Volvo afterburner experiment [43].

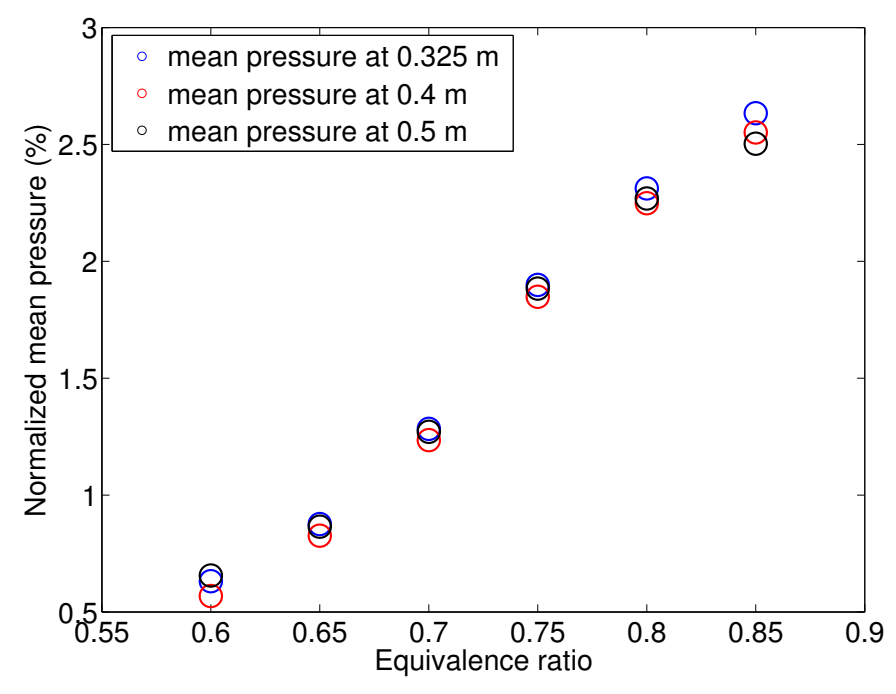

Figure 11.: Variation of mean pressure (as a percentage of the atmospheric pressure) with equivalence ratio

The instantaneous contours of the reaction progress variable, unsteady heat release rate and transverse velocity of the flame are shown in Fig. 12 at each equivalence ratio. The three columns in the figure correspond to reaction progress variable (first column), unsteady heat release (second column) and transverse velocity (third column). The six rows correspond to increasing equivalence ratios from top to bottom. Variations in the global flame structure can be seen as the equivalence ratio is increased. The flame exhibits a varicose structure at all equivalence ratios. At $\phi=0.60$ (first row), the flame is highly unsteady and strong symmetrical rollup of the upper and lower shear layers can be seen. At $\phi=0.65$ (second row), symmetrical vortex shedding is still present although the extent of large-scale flame wrinkling has decreased. At $\phi=0.70$ and $\phi=0.75$ (third and fourth rows respectively), the 
flame loses the large-scale unsteadiness that is present at lower equivalence ratios. The stabilizing effect caused by the large change in the density across the flame plays an important role in resisting the vortical rollup. At $\phi=0.80$ (fifth row), the flame becomes highly unsteady and a strong rollup can be seen downstream of the bluff-body flameholder. Further downstream, strong turbulent mixing occurs and exothermicity of the flame dominates. Similar behaviour is present for the flame at $\phi=0.85$ (bottom row), where large scale rollup of vortices in the shear layers induces a flow field that wraps the flame around these regions.
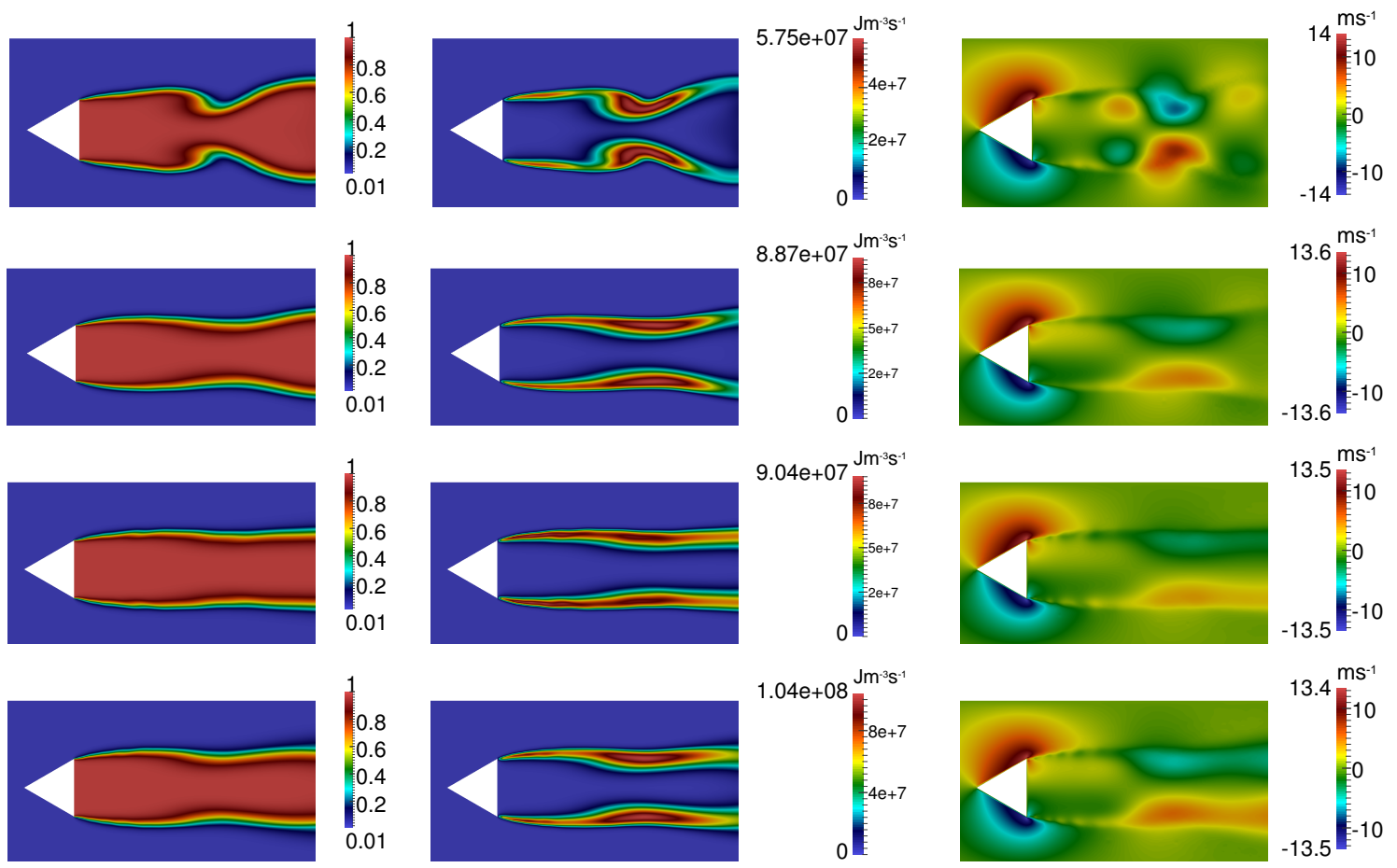

$13.4^{\mathrm{ms}^{-1}}$
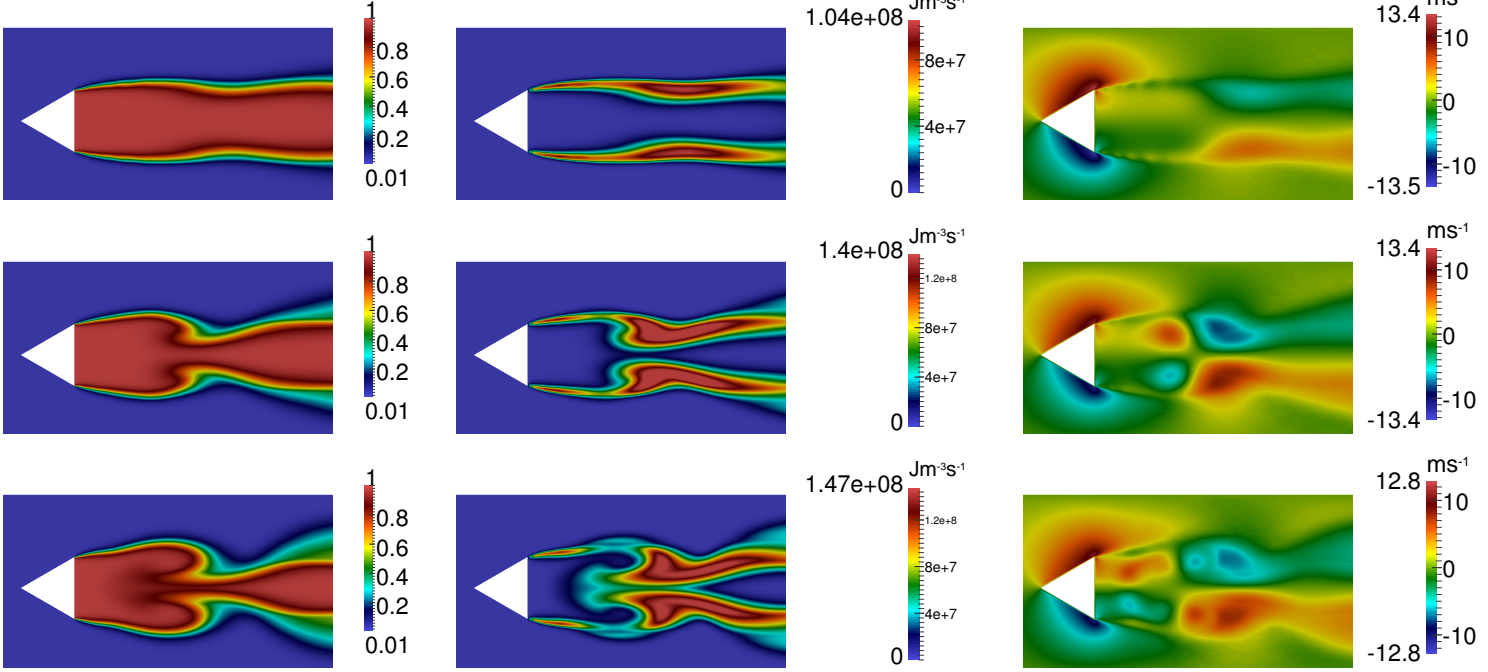

Figure 12.: Reaction progress variable (left), heat release rate (mid) and transverse velocity (right) at $\phi=0.6-0.85$ (rows $1-6$ ).

The magnitude of the global heat release increases with equivalence ratio over the range considered here. For all equivalence ratios, high unsteady heat release rate can be observed along the separated shear layer immediately downstream of the bluff-body. At low $(\phi=0.60)$ and high $(\phi=0.80$ and $\phi=0.85)$ equivalence ratios, high heat release is present in localized regions where an increase in the flame surface area corresponding to the rollup of the flame front occurs. At $\phi=0.65$, intense heat release can be seen in regions where the flame experiences high stretch. At intermediate equivalence ratios of $\phi=0.70$ and $\phi=0.75$, the unsteady heat release 

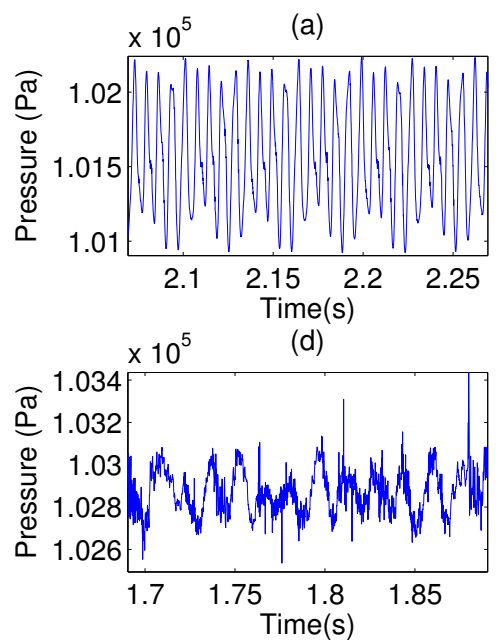
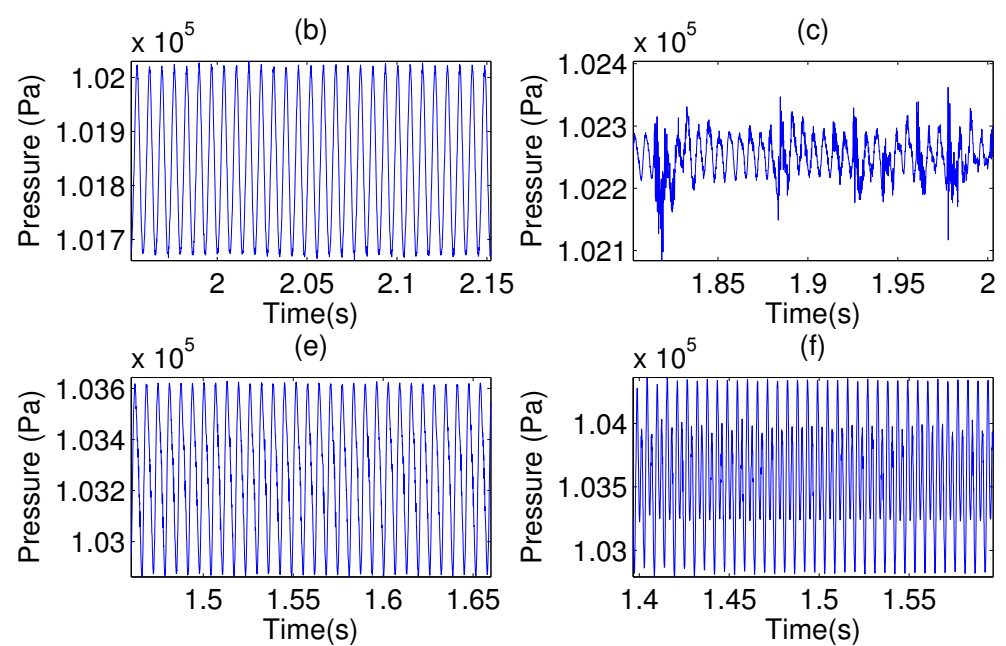

Figure 13.: Pressure time series at different equivalence ratios. (a) $\phi=0.6$, (b) $\phi$ $=0.65$, (c) $\phi=0.7$, (d) $\phi=0.75$, (e) $\phi=0.8$, (f) $\phi=0.85$

is almost uniform along the shear layer in the absence of large-scale variations of the flame surface area. Localized changes in the unsteady heat release are present immediately behind the recirculation zone. To examine the effect of coherent flow structures on the variations in flame structure, contours of transverse velocity are shown shown in the third column of Fig. 12. At $\phi=0.60$ (first row), regions of transverse velocity with alternating signs remain evident downstream of the bluffbody. This suggests hydrodynamic effects dominate where vortex shedding takes place along the mixing layer. Flame rollup and amplification of heat release occur in the regions where the magnitude of the transverse velocity is highest. This behaviour disappears at $\phi=0.65$ (second row). Instead, a localized region with high transverse velocity is found downstream of the recirculation zone. At $\phi=0.70$ and $\phi=0.75$ (third and fourth rows), small-scale variations in transverse velocity can be seen along the separated shear layers behind the flameholder. Further downstream, this effect diminishes. The magnitude of the transverse velocity increases but remains fairly uniform in the axial direction along the upper and lower shear layers. At $\phi=0.80$ and $\phi=0.85$ (last two rows), flame rollup occurs close to the bluff-body flameholder. Corresponding regions of transverse velocity with alternating signs are present in the vicinity of the recirculation zone and the heat release increases significantly.

The global Rayleigh index for 50 complete cycles was also computed for each case to establish the presence of thermoacoustic instabilties. According to the Rayleigh criterion, thermoacoustic instability relies on the phase of the pressure fluctuations being within 90 degrees of that of the heat release oscillations. The Rayleigh criterion is used to provide an indication as to whether thermoacoustic instability occurs. If this criterion is satisfied, acoustic energy is fed into the system from the combustion process. Provided that the acoustic energy gained exceeds the energy lost at the boundaries due to convection or viscous processes, the pressure amplitude will grow until nonlinear effects dominate. The Rayleigh index for each case was obtained by integrating the product of the normalized local heat release and pressure in space over the whole domain and in time over 50 cycles according to the expression $\int_{d t} \int_{d V} p^{\prime} q^{\prime} d V d t$. The computed global Rayleigh index was found to take a positive value when the equivalence ratio is at or above 0.8 . This provides 
an indication that the oscillation at low equivalence ratio is not strongly coupled to the acoustics and hence is due mainly to the hydrodynamics. Conversely, thermoacoustic coupling is likely to occur at higher equivalence ratios. This point will be discussed in greater detail below. Similar observation have been made previously by several authors $[5,38]$ in cases where nominally stable conditions exist at lower equivalence ratio and transition towards a thermoacoustically unstable condition occurs once a certain critical value of equivalence ratio is reached. This is also in agreement with the oscillatory behaviour reported in the Volvo afterburner experiment [43].

To demonstrate the limiting behaviour of the dynamical system, the time series of the pressure fluctuations when the oscillation has saturated is shown in Fig. 13. The location of the point where the pressure signal is recorded is at $x=1.2 w_{f}$ from the flameholder and $y=0.95 w_{f}$ from the centreline, where $w_{f}$ is the width of the bluff-body flameholder. This location corresponds to the shear layer region, and was selected slightly downstream of the flow separation region behind the bluff-body to prevent the pressure signal from being contaminated by noise, and ahead of the downstream wake to avoid numerical uncertainties with regard to the flow information in that region but still allows for the nonlinearities of the flame response to be captured. A quasiperiodic time series, which is common in many thermoacoustic systems [31] is apparent at $\phi=0.60$ (Fig. 13a). The pressure signal takes a sinusoidal form at $\phi=0.65$ (Fig. 13b) before translating into a lower amplitude and less coherent behaviour at intermediate equivalence ratios of $\phi=0.70$ (Fig. 13c) and $\phi=0.75$ (Fig. 13d). It reverts to a sinusoidal waveform with a single frequency when the equivalence ratio is increased to $\phi=0.80$ (Fig. 13e). At $\phi=0.85$ (Fig. 13f), the pressure time series retains its sinosoidal form but now contains a subharmonic of the fundamental frequency and therefore repeats itself every two cycles. This is a signature of a period-two oscillation.

The rich nonlinear behaviour of the flame is demonstrated in Fig. 14 where the PSD and phase portrait of the pressure fluctuations are shown. For the PSD, the base unit for pressure at $1 \mathrm{~Pa}$ is taken as the reference value for $\mathrm{dB}$ conversion. At $\phi=0.60$ (Fig. 14a), two peaks at incommensurate frequencies are present in the PSD at $141 \mathrm{~Hz}$ and at $113 \mathrm{~Hz}$. Sidebands also appear due to nonlinear interaction between these two independent modes. In the phase portrait, quasiperiodicity reveals itself as a torus. At $\phi=0.65$ (Fig. 14b), a distinct peak at $144 \mathrm{~Hz}$ and its harmonics are present. A closed phase trajectory suggests that the flame oscillates in a limit cycle. Transition from the quasiperiodic oscillation at $\phi=0.60$ to periodic at $\phi=0.65$ occurs via an inverse Neimark-Sacker bifurcation [70]. At $\phi=0.70$ (Fig. 14c) and $\phi=0.75$ (Fig. 14d), multiple spectral peaks can be seen in the PSD. The appearance of these frequencies is a consequence of the broadband excitations induced by the smaller scale vortical structures in the shear layers shown in Fig. 12 (column 3, row 3 and 4 ). The phase portrait reveals a fixed point attractor affected by noise. This indicates that combustion is steady and hence is "stable". At $\phi=0.8$ (Fig. 14e), the system bifurcates into a limit cycle with the dominant frequency occurring at $150 \mathrm{~Hz}$ with the presence of harmonics. Such a behaviour can be confirmed from the phase portrait where a distinct closed loop is present. As the equivalence ratio is increased to $\phi=0.85$ (Fig. 14f), two peaks at $156 \mathrm{~Hz}$ and $329 \mathrm{~Hz}$ with equal amplitudes appear in the PSD. In the phase portrait, the phase trajectory is closed with two loops, one corresponding to the oscillation at $156 \mathrm{~Hz}$ and another corresponding to $329 \mathrm{~Hz}$. The presence of double-loop structure is a characteristic of a period-two limit cycle. While the higher frequency is expected to be exactly twice the lower one for the period-two limit-cycle, this was not observed in the PSD. This is attributed to the limited spectral resolution in this study. To 
(a)

(b)
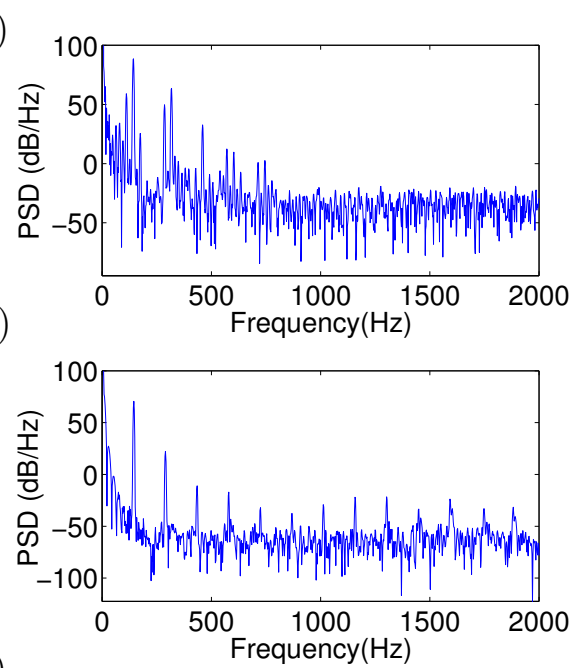

(c)

(d)

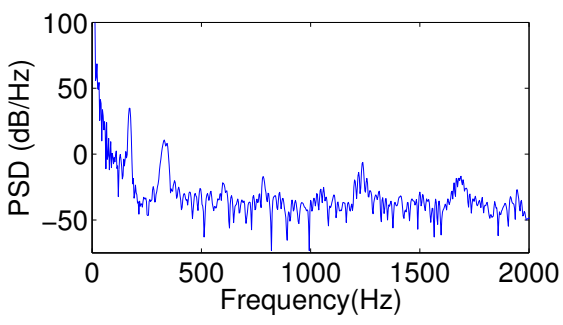

(e)

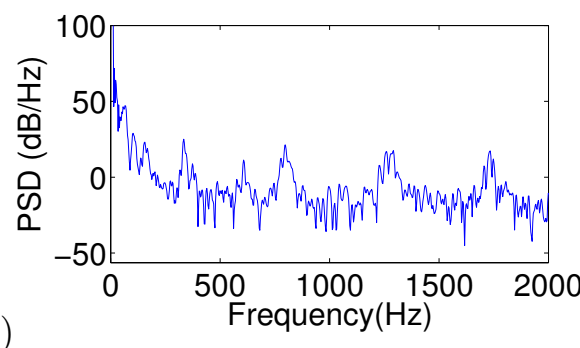

(f)
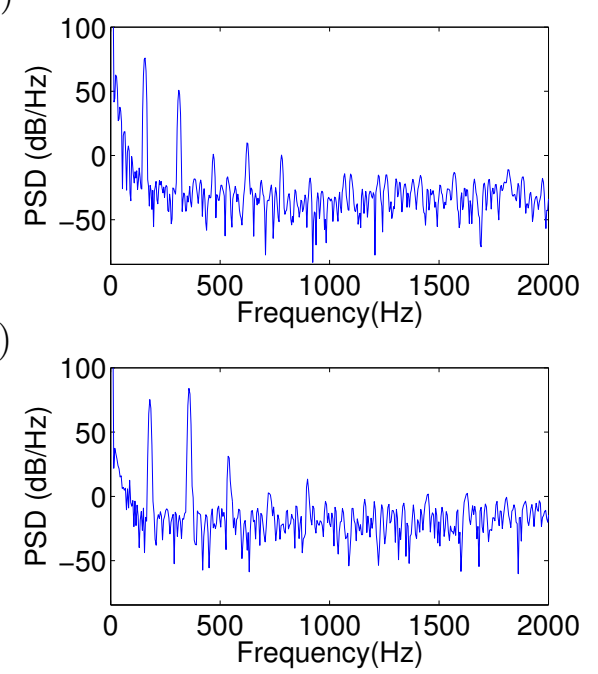
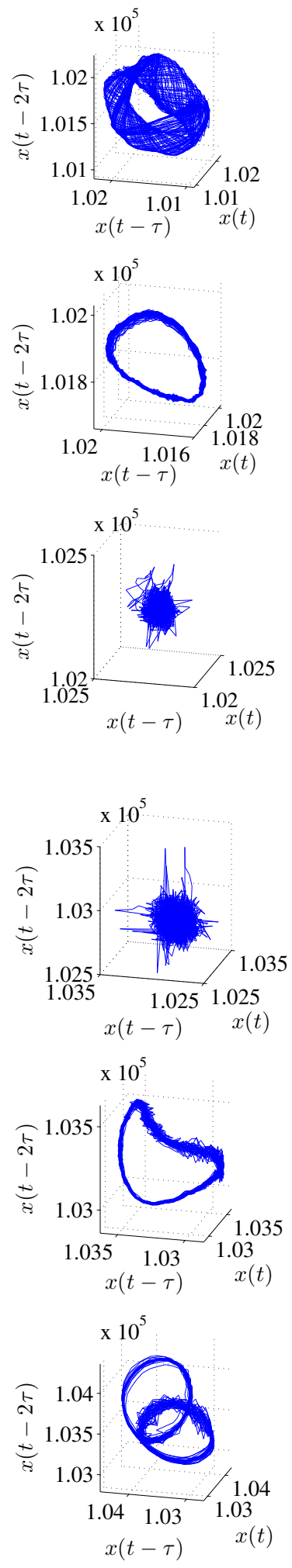

Figure 14.: (Left) PSD; and (right) phase portrait. (a) $\phi=0.6$, (b) $\phi=0.65$, (c) $\phi=0.7$, (d) $\phi=0.75$, (e) $\phi=0.8$, (f) $\phi=0.85$ 


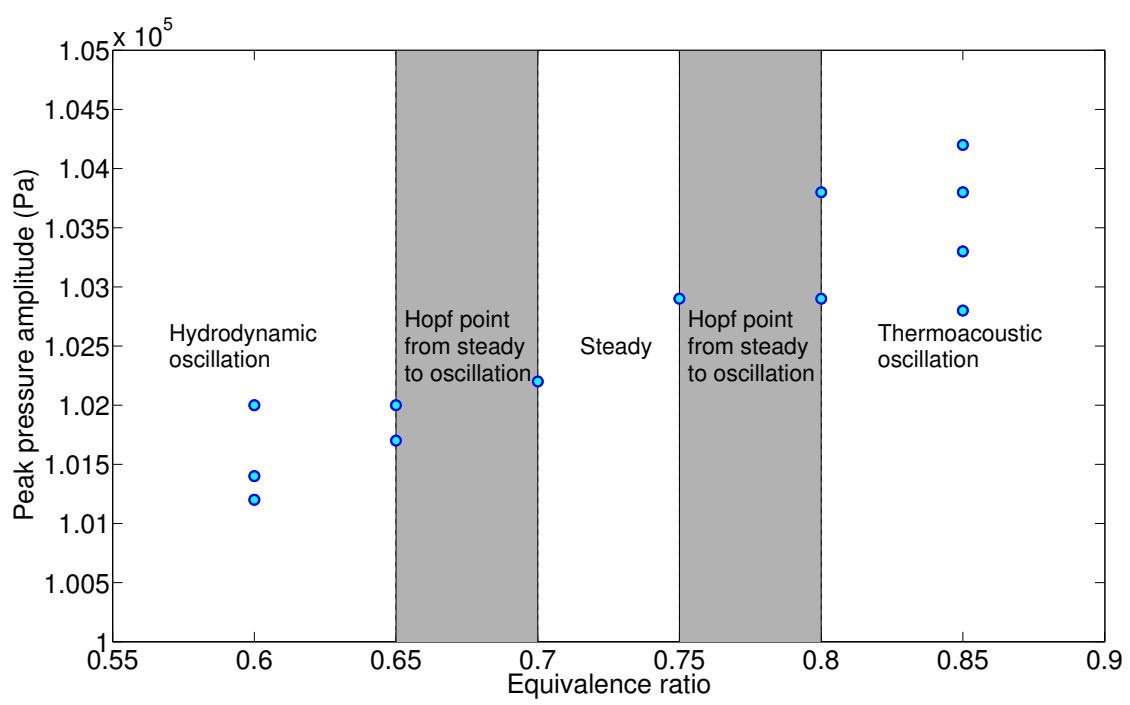

Figure 15.: Qualitative bifurcation diagram

verify the topological dimension of the attractor, the correlation dimension is also computed using the Grassberger-Procaccia algorithm [71]. The correlation dimensions are found to be consistent with the dynamical state identified from Fig. 14.

The global flame stability can be understood by plotting the peak amplitude of the pressure time series against equivalence ratio to produce a bifurcation diagram which is shown in Fig. 15. A further study would be useful to determine more precisely the nature and position of these Hopf points. The "stable" region lies within the range $\phi=0.70$ to $\phi=0.75$, and is labelled as "steady". Within this range the pressure fluctuation takes a single mean value and is affected only by random noise. There is no appreciable feedback to excite thermoacoustic oscillation. When the equivalence ratio is either decreased or increased from the steady region between $\phi=0.70-0.75$, the flame bifurcates to another state and a variety of dynamics have been observed. These correspond to multiple peaks (quasiperiodicity) at $\phi=0.60$, two peaks (limit cycle) at $\phi=0.65$, two peaks (limit cycle) at $\phi=0.80$ and four peaks (period-two) at $\phi=0.85$. At lower equivalence ratios $(\phi \leq 0.65)$, hydrodynamic oscillations occur when large-scale vortices are excited, resulting in strong perturbations of the flame. The hydrodynamic nature of this oscillation was observed in the Volvo afterburner experiment and is supported here by the negative Rayleigh index obtained at $\phi=0.60,0.65,0.70$ and 0.75 . At these equivalence ratios, the heat release fluctuations do not induce thermoacoustic oscillations. At higher equivalence ratios $(\phi \geq 0.80)$, the Rayleigh index is positive and the oscillation is caused by thermoacoustic feedback. During thermoacoustic oscillation, strong rollup of the flame surface occurs due to the presence of coherent structures in the separated shear layer downstream of the flameholder. The increase in heat release feeds energy to sustain the combustion oscillation, resulting in the observed limit cycles within this range of equivalence ratios. Since the simulations have been performed only at discrete values of equivalence ratio, the locations of the bifurcation points have been determined only within ranges denoted by the light grey regions in Fig. 15. 


\section{Conclusion}

Nonlinear dynamics of turbulent premixed bluff-body stabilized flames have been studied using turbulent combustion CFD with dynamical systems theory. The Volvo afterburner rig is used as the test case to elucidate the elaborate nonlinear dynamics, as well as changes in the global flame dynamics as the equivalence ratio is varied. A steady flame is observed between $\phi=0.70$ and $\phi=0.75$. Such dynamical behaviour is confirmed by the presence of a fixed point attractor in phase space and the lack of a dominant frequency in the PSD. The system bifurcates to an oscillating state between $\phi=0.65$ and $\phi=0.70$ and between $\phi=0.75$ and $\phi=0.80$. The system at $\phi=0.60$ and $\phi=0.65$ exhibits quasiperiodic behaviour (torus attractor) and a limit cycle (closed-loop attractor) respectively whereas the system at $\phi=0.80$ and $\phi=0.85$ corresponds to a limit cycle (closed-loop attractor) and period-two state (double closed-loop attractor) respectively. At these equivalence ratios, strong variations of the flame surface area are evident, corresponding to an increase in the heat release rate of the flame. Thermoacoustic instability is provoked at high equivalence ratios when the heat release and pressure fluctuations are in phase. At each bifurcation point, a strong change in the overall system behaviour is seen to occur. The observed dynamical behaviour plays a crucial role in identifying and understanding the operating conditions required for steady combustion. This approach can also serve as a first step in determining the key mechanisms that lead to the self-excited oscillation, and may therefore assist in developing control strategies to resist the onset of thermoacoustic instability.

\section{Acknowledgements}

The authors would like to acknowledge financial support from the Dorothy Hodgkin Postgraduate Award and Rolls-Royce Plc.

\section{References}

[1] Lord Rayleigh. The explanation of certain acoustical phenomena. Nature, pages 319-320, 1878.

[2] S. Candel. Combustion dynamics and control: progress and challenges. Proc. Combust. Inst., 29:1-28, 2002.

[3] A. P. Dowling. The calculation of thermoacoustic oscillations. J. Sound Vib., 180:557-581, 1995.

[4] A. P. Dowling and S. R. Stow. Acoustic analysis of gas turbine combustors. J. Propul. Power, 19:751-764, 2003.

[5] P. J. Langhorne. Reheat buzz: an acoustically coupled combustion instability. Part 1. Experiment. J. Fluid. Mech., 193:417-443, 1988.

[6] S. Ducruix, S. Candel, D. Durox, and T. Schuller. Theoretical and experimental determinations of the transfer function of a laminar premixed flame. Proc. Combust. Inst., 28:765-773, 2000.

[7] M. Zhu, A. P. Dowling, and K. N. C. Bray. Forced oscillations in combustors with spray atomizers. J. Eng. Gas Turb. Power, 124:20-30, 2002.

[8] C. A. Armitage, A. J. Riley, R. S. Cant, A. P. Dowling, and S. R. Stow. Flame transfer function for swirled LPP combustion from experiments and CFD. Proc. ASME Turbo Expo, GT2004-53820, Vienna, Austria, 2004.

[9] A. Gentemann, C. Hirsch, K. Kunze, F. Kiesewetter, and T. Sattelmayer. Validation of flame transfer function reconstruction for perfectly premixed swirl flames. Proc. ASME Turbo Expo, GT2004-53776, Vienna, Austria, 2004. 
[10] T. Schuller, D. Durox, and S. Candel. Self-induced combustion oscillations of laminar premixed flames stabilized on annular burners. Combust. Flame, 135:525-537, 2003.

[11] R. Balachandran, B. Ayoola, C. Kaminski, A. P. Dowling, and E. Mastorakos. Experimental investigation of the nonlinear response of turbulent premixed flames to imposed inlet velocity oscillations. Combust. Flame, 143:37-55, 2005.

[12] B. Ćosić, S. Terhaar, J. P. Moeck, and C. O. Paschereit. Response of a swirl-stabilized flame to simultaneous perturbations in equivalence ratio and velocity at high oscillation amplitudes. Combust. Flame, 162:1046-1062, 2015.

[13] N. Noiray, D. Durox, T. Schuller, and S. M. Candel. A unified framework for nonlinear combustion instability analysis based on the flame describing function. J. Fluid Mech., 615:139167, 2008.

[14] P. Palies, D. Durox, T. Schuller, and S. Candel. Nonlinear combustion instability analysis based on the flame describing function applied to turbulent premixed swirling flames. Combust. Flame, 158:1980-1991, 2011.

[15] D. Durox, T. Schuller, N. Noiray, and S. Candel. Experimental analysis of nonlinear flame transfer functions for different flame geometries. Proc. Combust. Inst., 32:1391-1398, 2009.

[16] S. Schimek, J. P. Moeck, and C. O. Paschereit. An experimental investigation of the nonlinear response of an atmospheric swirl-stabilized premixed flame. J. Eng. Gas Turb. Power, 133:17, 2011.

[17] K. R. Mcmanus, T. Poinsot, and S. M. Candel. A review of active control of combustion instabilities. Prog. Energy Comb. Sci., 19:1-29, 1993.

[18] S. Strogatz. Nonlinear Dynamics and Chaos. Westview Press, 1994.

[19] M. E. Kavousanakis, L. Russo, F. S. Marra, and C. Siettos. Homoclinic bifurcations in radiating diffusion flames. Comb. Theory Modelling, 17:40-52, 2013.

[20] P. Knoop, F. E. C. Culick, and E. E. Zukoski. Extension of the stability of motions in a combustion chamber by nonlinear active control based on hysteresis. Comb. Sci. Technol., 123:363-376, 1997.

[21] T. C. Lieuwen. Experimental investigation of limit-cycle oscillation in an unstable gas turbine combustor. J. Propul. Power, 18:61-67, 2002.

[22] Y. Huang and V. Yang. Bifurcation of flame structure in a lean-premixed swirl-stabilized combustor: transition from stable to unstable flame. Combust. Flame, 136:383-389, 2004.

[23] Y. Huang and V. Yang. Effect of swirl on combustion dynamics in a lean-premixed swirlstabilized combustor. Proc. Combust. Inst., 30:1775-1782, 2005.

[24] M. P. Juniper. Triggering in the horizontal Rijke tube: non-normality, transient growth and bypass transition. J. Fluid. Mech., 667:272-308, 2010.

[25] A. P. Dowling. Nonlinear self-oscillation of a ducted flame. J. Fluid. Mech., 346:271-290, 1997.

[26] A. A. Peracchio and W .M. Proscia. Nonlinear heat-release / acoustic model for thermoacoustic instability in lean premixed combustors. J. Eng. Gas Turb. Power, 121:415-421, 1999.

[27] K. Kashinath, I. C. Waugh, and M. P. Juniper. Nonlinear self-excited thermoacoustic oscillations of a ducted premixed flame: bifurcations and routes to chaos. J. Fluid Mech., 761:399-430, 2014.

[28] C. C. Jahnke and F. E. C. Culick. Application of dynamical systems theory to nonlinear combustion instabilities. J. Propul. Power, 10:508-517, 1994.

[29] J. D. Sterling. Nonlinear analysis and modelling of combustion instabilities in a laboratory combustor. Comb. Sci. Technol., 89:167-179, 1993.

[30] H. Gotoda and T. Ueda. Transition from periodic to non-periodic motion of a bunsen-type premixed flame tip with burner rotation. Proc. Combust. Inst., 29:1503-1509, 2002.

[31] L. Kabiraj, R. I. Sujith, and P. Wahi. Bifurcations of self-excited ducted laminar premixed flames. J. Eng. Gas Turb. Power, 134:1-7, 2012.

[32] A. Prasad and C. H. K. Williamson. The instability of the shear layer separating from a bluff body. J. Fluid. Mech., 333:375-402, 1997.

[33] L. Boyer, M. Provansal, and C. Mathis. Benard-von Khrmhn instability : transient and forced regimes. J. Fluid. Mech., 182:1-22, 1987.

[34] C. H. K. Williamson. Vortex dynamics in cylinder wake. Ann. Rev. Fluid Mech., 28:477-439, 1996.

[35] P. A. McMurtry, J. J. Riley, and R. W. Metcalfe. Effects of heat release on the large-scale structure in turbulent mixing layers. J. Fluid. Mech., 199:297-332, 1989.

[36] B. Emerson, J. O'Connor, M. Juniper, and T. Lieuwen. Density ratio effects on reacting bluff-body flow field characteristics. J. Fluid. Mech., 706:219-250, 2012. 
[37] K. C. Schadow, E. Gutmark, T. P. Parr, D. M. Parr, K. J. Wilson, and J. E. Crump. Large-scale coherent structures as drivers of combustion instability. Comb. Sci. Technol., 64:167-186, 1989.

[38] T. Poinsot, A. Trouve, D. Veynante, S. Candel, and E. Esposito. Vortex-driven acoustically coupled combustion instabilities. J. Fluid. Mech., 177:265-292, 1987.

[39] C. A. Armitage, R. Balachandran, E. Mastorakos, and R. S. Cant. Investigation of the nonlinear response of turbulent premixed flames to imposed inlet velocity oscillations. Combust. Flame, 146:419-436, 2006.

[40] S. Shanbhogue, D-H Shin, S. Hemchandra, D. Plaks, and T. Lieuwen. Flame sheet dynamics of bluff-body stabilized flames during longitudinal acoustic forcing. Proc. Combust. Inst., 32(2):1787-1794, 2009.

[41] C. Y. Lee and R. S. Cant. CFD investigation of turbulent premixed flame response to transverse forcing. Proc. ASME Turbo Expo, GT2013-94312, San Antonio, Texas USA, 2013.

[42] A. Sjunnesson, P. Henrikson, and C. Lofstrom. CARS measurement and visualization of reacting flow in a bluff body stabilized flame. AIAA/SAE/ASME/ASEE 28th Joint Propulsion Conference and Exhibit, July 6-8, Nashville, TN, 1992.

[43] A. Sjunnesson, S. Olovsson, and S. Sjöblom. Validation rig - A tool for flame studies. In ISABE Conference, Nottingham, UK, 1991.

[44] X.S. Bai. Modelling of turbulent reacting flows past a bluff body: assessment of accuracy and efficiency. Comp. Fluids, 23:507-521, 1994.

[45] P. Nilsson and X.S. Bai. Effects of flame stretch and wrinkling on co formation in turbulent premixed combustion. Proc. Combust. Inst., 29:1873-1879, 2002.

[46] C. Fureby. A computational study of combustion instabilities due to vortex shedding. Proc. Combust. Inst., 28:783-791, 2000.

[47] C. Fureby and C. Duwig. LES of premixed flame longitudinal wave interactions. Direct and Large-Eddy Simulation VI, 10:77-84, 2006.

[48] C. Fureby. Large eddy simulation of combustion instabilities in a jet engine afterburner model. Comb. Sci. Technol., 161:213-243, 2000.

[49] C. Nottin, R. Knikker, M. Boger, and D. Veynante. Large eddy simulations of an acoustically excited turbulent premixed flame. Proc. Combust. Inst., 28:67-73, 2000.

[50] I. Porumbel and S. Menon. Large eddy simulation of bluff body stabilized premixed flame. 44th AIAA Aerospace Sciences Meeting and Exhibit, Reno, Nevada, 2006.

[51] K. N. C. Bray, P. A Libby, and J. B. Moss. Unified modeling approach for premixed turbulent combustion - Part I : general formulation. Combust. Flame, 61:87-102, 1985.

[52] S. J. Brookes, R. S. Cant, I. D. J. Dupere, and A. P. Dowling. Computational modeling of self-excited combustion instabilities. J. Eng. Gas Turb. Power, 123:322-326, 2001.

[53] J. C. Rolon, S. Candel, P-H. Renard, and D. Thevenin. Dynamics of flame/vortex interactions. Prog. Energy Comb. Sci., 26:225-282, 2000.

[54] B. E. Launder, G. J. Reece, and W. Rodi. Progress in the development of a Reynolds-stress turbulence closure. J. Fluid. Mech., 68:537-566, 1975.

[55] B. J. Daly and H. H. Francis. Transport equations in turbulence. Phys. Fluids, 13:2634-2649, 1970.

[56] G. M. Abu-Orf and R. S. Cant. A turbulent reaction rate model for premixed turbulent combustion in spark-ignition engines. Combust. Flame, 122:233-252, 2000.

[57] R K Cheng and I G Shepherd. The influence of burner geometry on premixed turbulent flame propagation. Combust. Flame, 85:7-26, 1991.

[58] R. S. Cant, K. N. C. Bray, L. W. Kostiuk, and B. Rogg. Flow divergence effects in strained laminar flamelets for premixed turbulent combustion. Comb. Sci. Technol., 95:261-276, 1994.

[59] K. N. C. Bray and R. S. Cant. Some Applications of Kolmogorov's Turbulence Research in the Field of Combustion. Proc. Roy. Soc. A, 434:217-240, 1991.

[60] H. G. Weller, G. Tabor, H. Jasak, and C. Fureby. A tensorial approach to computational continuum mechanics using object-oriented techniques. J. Comp. Phys., 12:620-631, 1998.

[61] R. I. Issa. Solution of the implicitly discretised fluid flow equations by operator-splitting. $J$. Comp. Phys., 62:40-65, 1985.

[62] S. H. Johansson, L. Davison, and E. Olsson. Numerical simulation of vortex shedding past triangular cylinders at high Reynolds number using a $k-\epsilon$ turbulence model. Int. J. Num. Methods Fluids, 16:859-878, 1993.

[63] D. Ruelle and F. Takens. On the nature of turbulence. Commun. Math. Phys., 20:303-322, 1971.

[64] H. D. I. Abarbanel. Analysis of Chaotic Data. Springer-Verlag, New York, NY USA, 1996. 
[65] A. Sjunnesson, A. Olovsson, and E. Max. Measurements of velocities and turbulence in a bluff body stabilized flame. In Fourth International Conference on Laser Anemometry - Advances and Application, ASME Cleveland, US, 1991.

[66] E. Giacomazzi, V. Battaglia, and C. Bruno. The coupling of turbulence and chemistry in a premixed bluff-body flame as studied by LES. Combust. Flame, 138:320-335, 2004.

[67] C. Y. Lee. Numerical investigation of combustion instabilities in afterburners. PhD Thesis, University of Cambridge, 2015.

[68] D-H. Shin and T. Lieuwen. Flame wrinkle destruction processes in harmonically forced, turbulent premixed flames. J. Fluid. Mech., 721:484-513, 2013.

[69] B. Wegner, A. Maltsev, C. Schneider, A. Sadiki, A. Dreizler, and J. Janicka. Assessment of unsteady RANS in predicting swirl flow instability based on LES and experiments. Int. J. Heat. Fluid Flow, 25:528-536, 2004.

[70] L. K. B. Li and M. P. Juniper. Phase trapping and slipping in a forced hydrodynamically self-excited jet. J. Fluid. Mech., R5:1-7, 2013.

[71] P. Grassberger and I. Procaccia. Characterization of strange attractors. Phys. Rev. Lett., 50:346-349, 1994. 